\title{
Choice and response time processes in the identification and categorization of unidimensional stimuli
}

\author{
YVES LACOUTURE \\ Université Laval, Québec City, Québec, Canada \\ and \\ A. A. J. MARLEY \\ University of Victoria, Victoria, British Columbia, Canada
}

\begin{abstract}
Lacouture and Marley $(1991,1995,2001)$ have successfully modeled the probabilities of correct responses and the mean correct response times (RTs) in unidimensional absolute identification tasks for various stimulus ranges and stimulus/response set sizes, for individual and group data. These fits include those to a set of phenomena often referred to as end-anchor effects. A revised model, with the independent accumulator decision process replaced by a leaky competing accumulator decision process, fits the probabilities of correct responses and the full distributions of RTs in unidimensional absolute identification. The revised model is also applied successfully to a particular class of unidimensional categorization tasks. We discuss possible extensions for handling sequential effects in unidimensional absolute identification, and other extensions of the given class of categorization tasks that are of potential empirical and theoretical importance as a supplement to the study of multidimensional absolute identification tasks.
\end{abstract}

The ability to identify environmental features is of fundamental importance for cognition. For example, in unidimensional absolute identification tasks, observers must identify 1 out of $N$ unidimensional stimuli with an appropriate label. As Miller (1956) pointed out, a remarkable finding is that as $N$ approaches and then exceeds 7 , observers make an accelerating proportion of errors. In contrast, when observers make comparative judgments (i.e., as to whether one stimulus is greater than, equal to, or smaller than another stimulus) the number of stimuli that can be distinguished is much greater. This limit for unidimensional absolute judgments imposes severe practical restrictions on our ability to categorize unidimensional stimuli, with our ability to identify complex objects likely depending on other factors (e.g., semantics) in addition to their multidimensional structure. More generally, as Shiffrin and Nosofsky (1994) argued in an

This work was supported by Natural Sciences and Engineering Research Council of Canada (NSERC) Discovery and Equipment Grants to Y.L. and A.A.J.M., and by a team grant from the Fonds pour la formation de Chercheurs et l'Aide à la Recherche du Québec (FCAR) to Y.L. and A.A.J.M. The second author especially thanks the Hanse Wissenschaftskollegg for its fellowship, in all senses of the word. Thanks to Bruce Schneider and two anonymous reviewers for their detailed, careful reviews of an earlier version of the manuscript. Correspondence concerning this article should be addressed Y. Lacouture, École de Psychologie, Université Laval, Québec, PQ, G1K 7P4 Canada (e-mail: yves.lacouture@psy.ulaval.ca).

Note-This article was accepted by the previous editorial team, headed by Neil Macmillan. issue of Psychological Review in which the significance of numerous classic papers, including Miller's, was reassessed, "absolute identification has captured the imagination . . . not only because the empirical results are so startling but also because [they] provide perplexing problems for classic psychophysical models" (p. 358). There is extensive empirical literature, both "classical" and modern, on (unidimensional) absolute identification, and in recent years sophisticated attempts to model the empirical results have been made in rapidly growing numbers. Important empirical contributions include works by Ward and Lockhead (1970, 1971), Luce, Green, and Weber (1976), Weber, Green, and Luce (1977), Ward (1979), Luce, Nosofsky, Green, and Smith (1982), Lockhead (1984), Mori (1989), Ashby and Lee (1991), and, most recently, Parker, Murphy, and Schneider (2002). These contributions (with the exception of the final one) and other, earlier results are summarized in Luce (1986, chap. 10), Lacouture and Marley (1995), Lacouture (1997), and Lacouture and Lacerte (1997). Important theoretical works include the context-based Thurstonian approach of Berliner, Braida, and Durlach, beginning with Durlach and Braida (1969) and continuing with a long series of excellent theoretical and empirical studies (Berliner \& Durlach, 1973; Berliner, Durlach, \& Braida, 1977; Braida \& Durlach, 1972; Braida et al., 1984); Laming's (1984, 1997) relative judgment model; Treisman and Williams's (1984) model based on criterion setting theory; the attention band approach of Luce and colleagues (Luce, 1986; Luce et al., 1976; Luce et al., 1982; Weber, Green, \& Luce, 1977); 
Ashby, Lee, and Balakrishnan's (1992) comparison of the biased choice model and the decision-bound model; and Nosofsky's (1997) exemplar-based random-walk model of absolute identification. Regrettably, there is no recent integrative review of this empirical and/or theoretical literature, and we do not attempt to present one here.

In our view, there is not yet a single well motivated model that adequately handles all the relevant data. For instance, there is no model that simultaneously predicts both the "classical" data on probability correct (PC), information transmitted, sequential effects, and so forth, and recent data on full response time (RT) distributions for individual participants. Our own earlier model did not deal with sequential effects, nor did it adequately predict full RT distributions. The present article rectifies the situation with respect to full RT distributions and addresses the question of how the revised model might be extended to handle sequential effects. We fully realize that sequential effects are important and might, in fact, form the basis of a complete model. However, we made the strategic choice to concentrate first on other major effects; of course, to defend this strategic choice we must be able to revise and/or extend our model in a reasonable way to include such sequential effects. We also apply the model to a particular (binary) categorization task for unidimensional stimuli that, as will be discussed later, has considerable potential for extension to related multidimensional tasks.

We now introduce our notation for the basic (unidimensional) absolute identification task, describe various data, and introduce the revision of our model that enables us to handle full RT distributions.

A typical unidimensional absolute identification experiment involves a set of $N$ stimuli that vary along some physical dimension. On each trial, 1 of the $N$ stimuli is presented, and the participant's task is to select the previously specified "correct" response for that stimulus. RT is usually measured by the activation of a key on a keyboard, with each key being associated with exactly one of the numerals 1 through $N$ and with the usual order of the numerals being in agreement with the psychological "magnitude" of the stimuli to be identified. Recently, voice-activated keys have been used in place of keyboards, and this is the case here. One major phenomenon is that no matter how widely spread the stimuli are on the relevant sensory dimension, people are usually able to correctly identify only about 7 of them. Although our own empirical and theoretical work includes study of this basic result, we have gone much further and studied a second set of phenomena, related to choice and choice times, that are variously referred to as the end-anchor effect, the bow effect, and sometimes the serial position effect (Lacouture, 1997; Lacouture \& Marley, 1991, 1995). These terms refer to the fact that accuracy decreases and RT increases as one moves away from the stimuli at the end of the presented range; the effects also become more pronounced for larger set sizes.
An important empirical and theoretical issue is whether or not such end-anchor effects might be due simply to the fact that the numerical responses are bounded at the lower and upper ends of their range, and/or to the fact that keyboards usually have "end" keys. Partial evidence that should alleviate such concerns is that signal detection analyses of the choice probabilities in unidimensional absolute identification show better discrimination (larger $d^{\prime}$ ) for stimuli (responses) toward the end of the range (see, e.g., Durlach \& Braida, 1969; Lacouture \& Marley, 1995). Here, we report data on unidimensional absolute identification using a voice key as the response mode, that are essentially identical to data obtained using a standard keyboard.

We also explore related issues concerning both keyboards and voice keys that arise from the desire to study the absolute identification of multidimensional stimuli. We have not been able to design a satisfactory response mode for stimuli/responses of two (or more) dimensions that allows us to measure RT without confounds due to factors such as whether the responses for each dimension are reported sequentially. We now discuss this issue as a motivation for the study of a particular class of categorization experiments that we believe circumvents many of these issues, and that is also of interest in its own right.

In a typical absolute identification task with unidimensional stimuli, the stimuli and responses are compatible in the sense that the ordering of the stimuli from the smallest to the largest magnitude corresponds to the ordering of the responses. Typically, stimuli are assigned the response numerals " 1, , " 2, , " 3, , . . , " $N$ " in increasing order corresponding to the increasing magnitude of the stimuli. Also, with motor responses the keyboard layout is usually such that each response (" 1 " to " $N$ ") requires the same motor displacement; for instance, as in our own experiments, the response keys are placed in a semicircle with each key the same distance from a central START key that is used by the participant to initiate a trial.

Both practical (empirical) and conceptual (theoretical) problems arise in extending the study of absolute identification to multidimensional stimuli, especially when one requires the accurate measurement of RTs. Here, we discuss briefly the empirical issues and present one approach to their resolution; later, we discuss theoretical interpretations of the data obtained using this approach.

Consider a set of "2-D" stimuli, such as acoustic stimuli that vary in intensity and frequency with, say, four levels of intensity crossed with four levels of frequency. The natural mapping of each of the 16 stimuli to each of the two-digit labels " 1,1, , " 1,2, , . . . " 4,4 " gives stimulusresponse compatibility, yet more than one response-for example, " 1 " followed by " 2 "- - is then necessary to identify each stimulus, which creates problems for the accurate measurement of RTs. For instance, a $4 \times 4$ array of keys is compatible with the stimulus design above, but then there is no natural placement of the resting key (START button) that is equidistant from all 16 response 
keys. Parallel issues arise with the use of spoken responses (recorded by a voice key), except in the very special case in which the stimuli already have "names." We have carried out various exploratory studies of the absolute identification of 2-D and 3-D stimuli using the types of response measures mentioned above; participants found the tasks very difficult, and the RTs were highly variable, with complex sequential dependencies.

This exploratory work has led us to a different approach using categorization. Continuing with the example above, instead of having each stimulus associated with a bivariate (two-digit) response, we associate each of the 16 stimuli with one of two (response) categories, Category A and Category B, with the "natural" mapping from stimuli to response categories as shown in Table 1 and with the (manual) response for each category being associated with a different key on a keyboard. Thus, we require the use of only two response keys (plus a START key), which is standard in many psychophysical experiments that measure RTs, and, thus, we have eliminated the use of bivariate responses. Nonetheless - and this is equally important - the design of the categorization task is such that stimuli that are nearest neighbors in the stimulus space are assigned to different categories, and, thus, the precise value of each stimulus on each of its physical dimensions is relevant to performing the categorization task. ${ }^{1}$ It is the latter requirement that gives the link between the identification and categorization tasks. Later, we discuss some perceptual/cognitive processes that might be involved in performing such a categorization task, and the relevance of the data from such a task to our motivating interest in multidimensional absolute identification. For now, we discuss the unidimensional version of the categorization task.

Consider a set of 10 unidimensional stimuli, such as lines varying in length. Let " 1 " denote the shortest line and " 10 " the longest line, with the remaining lines and associated responses ordered in agreement with the order of these stimulus-response pairs. Now, carry out a categorization task in which the participant is told to respond "(Category) A" whenever Stimulus 1, 3, 5, 7, or 9 is presented and "(Category) B" whenever Stimulus 2, 4, 6, 8, or 10 is presented. We present results, for an individual participant and over participants, that show close agreement between the data (PC and full correct RT distributions) for such a unidimensional categorization task and

Table 1

Response Matrix for the Proposed Two-Category Categorization Task With 2-D Auditory Stimuli

\begin{tabular}{ccccc} 
Level of & \multicolumn{4}{c}{ Level of Intensity } \\
\cline { 2 - 5 } Frequency & 1 & 2 & 3 & 4 \\
\hline 1 & A & B & A & B \\
2 & B & A & B & A \\
3 & A & B & A & B \\
4 & B & A & B & A
\end{tabular}

Note-The entries in the matrix represent the correct response for each of the 16 stimuli (four levels of intensity by four levels of frequency). for the unidimensional absolute identification task with the same stimuli. These data support our suggestion that the categorization task has close relations to the absolute identification task. We provide excellent fits of our revised model to both sets of data, and in the Summary and Future Directions section we consider the potential importance of a generalization of this categorization approach as an alternative to multidimensional absolute identification. Nonetheless, the alternative categorization tasks provide choice and RT data of direct relevance to the research questions motivating the study of the absolute identification tasks.

In summary, Lacouture and Marley (1995) successfully modeled the relevant accuracy data and the general pattern of the mean correct RT data in absolute identification. That model has also been extended to special cases of unidimensional categorization - that is, to the categorization of unidimensional stimuli (Lacouture, Li, \& Marley, 1998). However, to date, the model has not been applied to complete RT distributions or to the general case of unidimensional categorization. Lacouture and Marley (2001) summarize preliminary results of the revised model presented in this article, showing that it provides a good account of the dynamics of the decision process and, consequently, of the full distributions of RTs.

Both our original and revised mapping models of unidimensional absolute identification ${ }^{2}$ are implemented as a three-layer connectionist feedforward network with linear units (see Figure 1, top panel). The sensory input is mapped to a (bounded) unidimensional (scalar) internal representation, which is in turn mapped via a theoretically motivated set of linear functions to an $\mathrm{N}$-dimensional output vector. A key feature of the model is the specific set of weights and biases used in the linear mappings of the internal scalar representation to the $\mathrm{N}$-dimensional output vector (see additional details below). This output vector in turn provides the input to the decision process. In our earlier work, the decision process involved a set of $N$ independent linear accumulators, with the first accumulator to reach a common prespecified threshold triggering the associated overt response. The addition of Gaussian noise at each of the input, internal, and output levels of the process enabled the mapping model, with a single set of parameter values, to reproduce core characteristics of identification and categorization data. These include overall performance limitations and bow effects in terms of both PC and mean correct RT. The present work demonstrates that the combination of our basic mapping model with a leaky competing accumulator decision process (Usher \& McClelland, 2001) provides a framework that successfully extends our previous results to fit the full distributions of RTs in identification tasks and certain special categorization tasks, as opposed to merely fitting mean correct RTs.

\section{The Mapping Model}

Lacouture and Marley's (1995) mapping model of absolute identification is developed in terms of a neural 

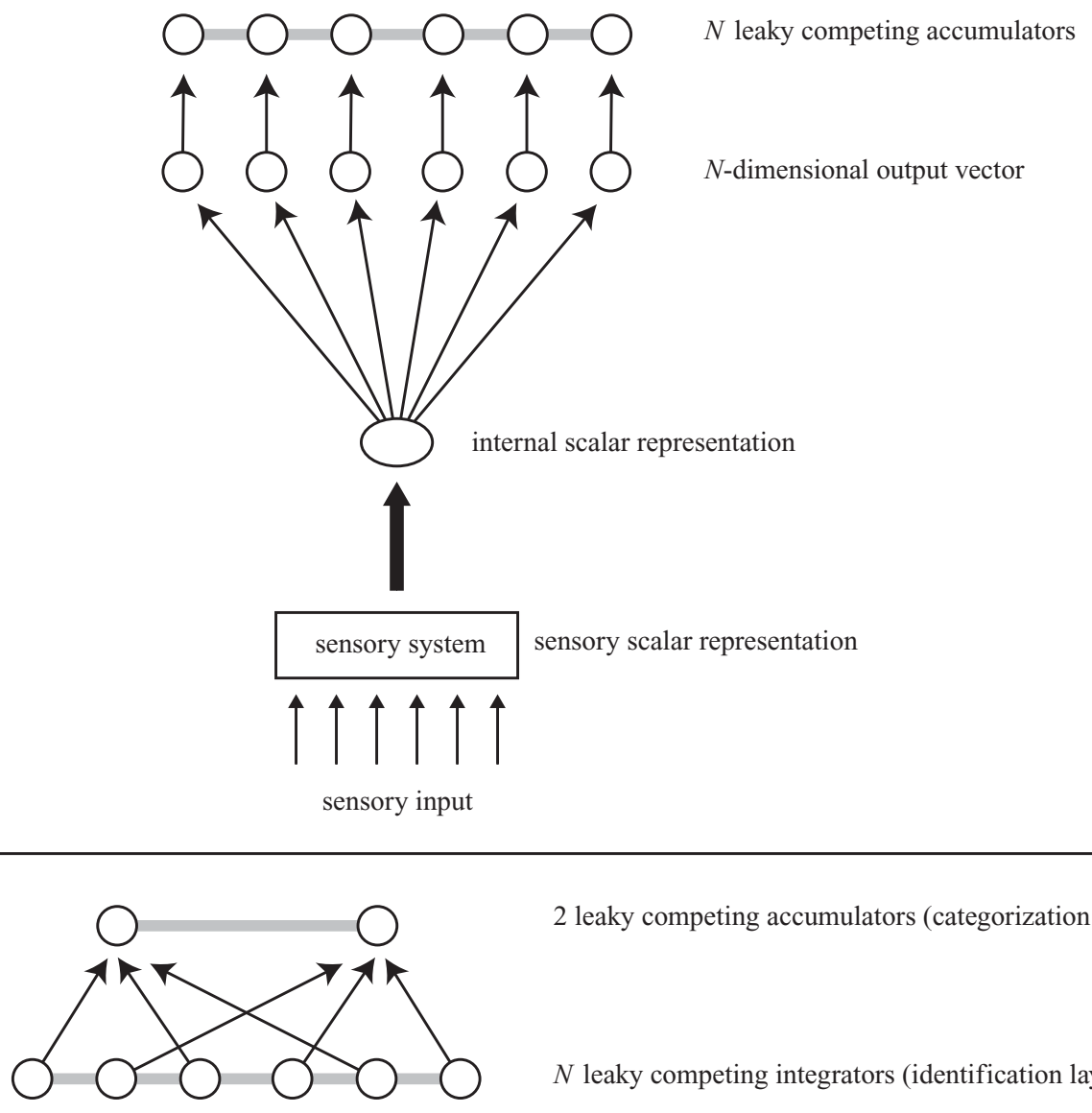

2 leaky competing accumulators (categorization layer)

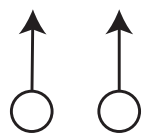

$\uparrow \uparrow$

$\uparrow$

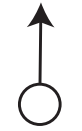

$N$ leaky competing integrators (identification layer)

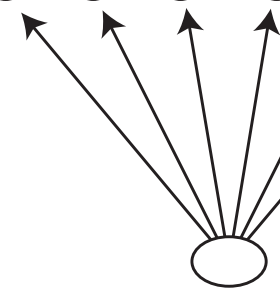

internal scalar representation

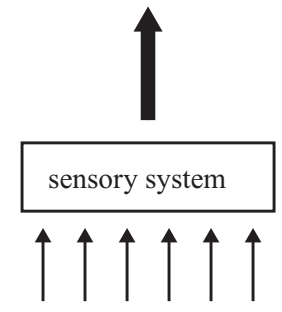

sensory input

Figure 1. The leaky competing accumulator process: architecture to model identification tasks (top panel) and categorization tasks (bottom panel).

network consisting of a sensory input system (a single input unit), one hidden unit, and multiple output units. Important assumptions of the model are that the activity in the hidden unit can take any real value in a restricted range, and that the sensory input is transformed into that range. In the following, we assume that range to equal 1; other values would simply lead to a rescaling of other parameters in the model. Now let $\mathrm{S}_{N}(i)$ be the psychological 
intensity of stimulus $i, i=1, \ldots, N$, in an absolute identification experiment with $N$ stimulus-response pairs. We assume that there is a lower anchor $\mathrm{S}_{N}(\mathrm{~L})$, with $\mathrm{S}_{N}(\mathrm{~L}) \leq$ $\mathrm{S}_{N}(1)$, and an upper anchor $\mathrm{S}_{N}(\mathrm{U})$, with $\mathrm{S}_{N}(N) \leq \mathrm{S}_{N}(\mathrm{U})$, such that the (mean) input to the hidden unit is given by

$$
\left[\frac{\mathrm{S}_{N}(i)-\mathrm{S}_{N}(\mathrm{~L})}{\mathrm{S}_{N}(\mathrm{U})-\mathrm{S}_{N}(\mathrm{~L})}\right]
$$

(Note that later our detailed assumptions regarding the position of the lower and upper anchors will require that both the lowest and the highest stimuli are sufficiently far from the ends of the psychological range; this condition is satisfied in the experiments reported in this article.)

Furthermore, we assume that on a given trial multiple samples of the presented stimulus are collected by a participant and are then (naturally) interpreted as multiple samples in the network. We also assume, in line with traditional Thurstonian models, that the psychological representations vary across samples - that is, they are "noisy." Specifically, we suppose that the $k$ th noisy sample value, $k=1,2,3, \ldots$, when stimulus $i$ is presented on the current trial, has value $\mathrm{S}_{N}(i)+N^{k}$, with $N^{k}$ normal with mean zero and standard deviation $(S D) \gamma$. The input to the hidden unit for the $k$ th noisy sample is then

$$
H_{N}^{k}(i)=\left[\frac{\mathrm{S}_{N}(i)-\mathrm{S}_{N}(L)+N^{k}}{\mathrm{~S}_{N}(U)-\mathrm{S}_{N}(\mathrm{~L})}\right]+H^{k},
$$

where $H^{k}$ is a normal random variable with mean zero and $S D \eta$. Note that this representation assumes that $\mathrm{S}_{N}(\mathrm{~L})$ and $\mathrm{S}_{N}(\mathrm{U})$ are represented accurately (i.e., without noise). We could add noise to these anchor values, but for the large range cases on which we focus, such noise - on the basis of samples of $N^{k}$ - has little effect on the overall results.

We now consider a special case of Equation 1 that is appropriate for the conditions of our later experiments. We assume that the stimuli have equal spacing on the psychological dimension, ${ }^{3}$ that the lower anchor is half that spacing below the lowest stimulus, and that the upper anchor is half that spacing above the highest stimulus. These assumptions with the representation of Equation 1 lead to the result that the mean activity levels in the hidden unit for stimulus $i$ is $(2 i-1) / 2 N$. In particular, stimulus 1 is mapped to the mean value $1 / 2 N$, stimulus $N$ is mapped to the mean value $1-1 / 2 N$, and all mean stimulus values are mapped into the interval $[0,1]$.

The output activation for output unit $j, j=1, \ldots, N$, is given by

$$
O_{N}^{k}(j \mid i)=\left[a(j) H_{N}^{k}(i)+b(j)\right]+D^{k}(j),
$$

where $D^{k}(j), j=1, \ldots, N$ is a normal random variable with mean zero and $S D \varepsilon$ and $a_{N}(j), b_{N}(j), j=1, \ldots, N$ are weights and biases connecting the single hidden unit to the layer of output units. Values of the weights and biases are uniquely determined for each set size by the mapping solution chosen by Lacouture and Marley (1995) (in which two solutions were developed, only one of which was appropriate for available absolute identification data). The general form of the linear mappings in Equation 3 follows from a theoretical solution of the socalled encoder problem (Lacouture \& Marley, 1995), which is equivalent, in our formulation, to the absolute identification task. In fact, this solution of the encoder problem (i.e., the absolute identification problem) involves linear mappings (Equation 3) followed by a winner-take-all decision process (i.e., the leaky competing accumulator model, below). It is important to note that the values of the weights and biases, $a_{N}(j), b_{N}(j)$, $j=1, \ldots, N$, for the case of equally spaced stimuli are determined by three parameters that have the same values for all $N$, plus the values of $N$ and $j=1, \ldots, N$.

Finally, within a trial the activation of each output unit is accumulated in an associated accumulator. The contents of each accumulator are assumed, for now, to be set to zero at the beginning of each trial ${ }^{4}$; thus, for stimulus $i$ and intratrial sample $k$, activation in the accumulator associated with output $j$ is

$$
A_{N}^{k}(j \mid i)=A_{N}^{k-1}(j \mid i)+O_{N}^{k}(j \mid i) \text { for } k \geq 1,
$$

where $O_{N}^{k}(j)$ is the current activation of output unit $j, \mathrm{~A}_{N}^{k}$ $(j)$ is the integrated activation after $k$ intratrial samples, and $A_{N}^{0}(j \mid i)=0$. In the basic mapping model (Lacouture \& Marley, 1995), each independent linear accumulator has the same threshold for "firing," and the response associated with the first accumulator to reach its threshold is made; the threshold is independent of set size and signal value. Note that there is no noise in Equation 4. We later present the full form of the model that generalizes Equation 4 in several ways, including the addition of noise.

Also, later in this article we present data for the following unidimensional categorization task. We map each of the 10 stimuli, $\mathrm{S}_{N}(i), i=1, \ldots, 10$, uniquely into one of two response categories, $\mathrm{A}$ and $\mathrm{B}$, with stimuli $\mathrm{S}_{N}(i)$, $i=1,3,5,7$, and 9 associated with Category A and Stimuli $\mathrm{S}_{N}(i), i=2,4,6,8$, and 10 associated with Category B.

\section{The Leaky Competing Accumulator Decision Process}

As we stated earlier, replacing the set of independent linear accumulators with a common threshold with the leaky competing accumulators extends the mapping model in a plausible way that can then fit full RT distributions. The basic mathematics of such a leaky competing accumulator decision process (developed by Usher \& McClelland, 2001 ) is presented in the Appendix. We now apply those results to $N$-alternative absolute identification. Let $O(j \mid i), j=1, \ldots, N$ be the outputs of our mapping model when stimulus $i$ is presented, which then become the input to accumulators $j, j=1, \ldots, N$. The current activation level in each accumulator decays (leaks) over time with parameter $\lambda$; there is self-amplification in each accumulator with parameter $\alpha$ and an inhibitory signal from the 
other accumulators with parameter $\beta$. Although the dynamics of the decision process are nonlinear, theoretical and simulation results show that, for $O(j \mid i)>0$, we can consider the parameters $\lambda$ and $\alpha$ to be additive in their effects. Therefore, we replace the value $(\lambda-\alpha)$ with a net leakage parameter $l$, so activation decays when $l>0$ and self-amplifies when $l<0$.

In the simulations, we used a variant of Equation A3 of the Appendix, with $\Delta$ being a parameter that can be set to various values corresponding to the desired fineness of the simulation relative to real time (in milliseconds). Thus, we have

$$
\begin{aligned}
& d x_{i}=\Delta\left[\rho_{i}-l x_{i}-\beta \sum_{i^{\prime} \neq i} x_{i^{\prime}}\right] \frac{d t}{\tau}+\xi_{i} \sqrt{\frac{d t}{\tau}}, \\
& x_{i} \rightarrow \max \left(x_{i}, 0\right) .
\end{aligned}
$$

With larger values of $\Delta$, the activation in each accumulator grows faster, giving a coarser representation of the dynamics of the process and shorter computational times; smaller values of $\Delta$ produce slower accrual and a finer resolution in the simulated process, but longer computational times. ${ }^{5}$ Although the simulations reported in this article run very efficiently on modern computers, we set $\Delta$ so that the resolution of the simulated process is fine enough to allow a good representation of the distribution of RTs, and yet, when the model is fitted to empirical data, it allows us to perform parameter search in a reasonable amount of time. The parameter $\Delta$ might also be used to implement a speed-accuracy tradeoff mechanism. In the present implementation of the model, the inputs, $\rho_{I}$, to the leaky competing accumulators (in the identification layer) are the output values of the mapping solution. The results of the simulated process depend on both the parameters of the mapping solution and the parameters of the leaky competing accumulators; this point is discussed below.

Returning to Equation 5, note the nonlinearity introduced in the second line, where the value of $x_{i}$ is set to zero if $x_{i} \leq 0$. Also, note that Equation 5 with $l=\beta=0$ and $x>0$ reduces to a set of independent linear accumulators (Lacouture et al., 1998; Lacouture \& Marley, 1995). There is an important difference between such a set of simple accumulators and the general leaky competing accumulator process: Because of the inhibitory processes in the latter when $l>0$ and $\beta>0$, the activations converge toward zero for all but a single unit. Also, the asymptotic activation in the remaining active unit reaches an equilibrium level when the net input to that unit equals the sum of the leakage signals. This corresponds to an implementation of a winner-take-all network in which a dynamic competitive process asymptotically yields activation in a single unit (Yuille \& Geiger, 1995).

\section{Dynamics of the Decision Process}

We simulated the dynamics of the decision process in the absolute identification task with 10 stimuli. We assume that each of the 10 accumulators has zero activation before the stimulus is presented and that $x_{j}=0$ for all $j$ at time zero (see note 4 and the Summary and Future Directions section). At each time step, Equation 5 is used to compute the change in activation, with the external input $\rho_{i}$ to each accumulator given by the output from the relevant mapping process. This value is given, for each accumulator in each time step, by Equation 3. Because of the noise at the input and hidden levels of the mapping model, the input value $\rho_{i}$ changes at each time step.

As has already been stated, with $l>0$ and $\beta>0$ the inhibitory signal makes the activation in every accumulator but one tend toward zero over time, and the maximum activation of a given accumulator is bounded. A state of equilibrium is reached when $\rho_{i}=l x_{i}$ (see Equation 5), with the activation of the winning unit fluctuating around this level. Also, at equilibrium, because there is only one active unit, the inhibitory signal fed to the winning unit is null:

$$
\beta \sum_{i^{\prime} \neq i} x_{i^{\prime}}=0 .
$$

Larger values of $l$ give lower values of the equilibrium activation point, and larger values of $\beta$ give larger inhibition, resulting in the "losing" units' dropping out faster and, thus, a shorter time for a single unit to win the race.

As we stated above, the dynamics of the decision process depend, on the one hand, on the specific values of $l$ and $\beta$ and, on the other hand, on the input values $\rho_{i}$, which in our implementation depend on the mapping solution and the noise parameters. Usher and McClelland (2001) have illustrated that, in a two-unit process, the competition among accumulators and the dynamics of the process depends on the difference in the level of activation fed to each of the two accumulator units. Smaller differences imply larger competition, a longer decision (response) time, and higher probability of error. We find parallel results in our simulation of absolute identification. When using the output of the mapping model as an input to the leaky competing decision process, the differences in output activation across the output units of the mapping model determines, in part, the dynamic of the decision process. Note that the mapping solution implies larger differences in output values (across output units) for stimuli located toward the ends of the range, and this will generally yield shorter RTs and higher PCs for these stimuli.

\section{EXPERIMENT 1}

\section{Method}

Participants. The participants were 12 undergraduate students, each of whom received $\$ 40$ (Canadian). All the participants reported having normal or corrected-to-normal vision, and none reported having a motor handicap.

Apparatus and General Procedure. The experiment was conducted in a dimly lit, sound-attenuated chamber. An MS-DOS-286 computer running Micro Experimental Laboratory software (MEL; Schneider, 1988) was used for stimulus presentation and response recording. Stimuli were presented within an angular distance of $10^{\circ}$ on a VGA screen (Fujikama, Modulux III with $640 \times 480$ pixels and 28 pixels $/ \mathrm{cm}$ ) located approximately $120 \mathrm{~cm}$ away from the participant. The computer screen was viewed through a rectangular 
opening of approximately $30 \times 25 \mathrm{~cm}$ made in a black cardboard frame of $1 \times 1 \mathrm{~m}$. In this setting, and under the low light condition of the experimental chamber, the edge of the computer screen could not be easily perceived. Stimulus presentation was synchronized with the vertical retrace of the screen.

Each of the participants performed identification and categorization of visual stimuli under two response conditions (manual and vocal). The two factors (task and type of response) defined four conditions in a $2 \times 2$ factorial design (manual identification, manual categorization, vocal identification, and vocal categorization). In the manual response conditions, responses were collected using a custom-made keyboard with 11 buttons. On this keyboard, 1 button, labeled "START," is located at the center of the 10 other buttons positioned in a semicircle in such a way that the START button is equally distant $(101 \mathrm{~mm})$ from each of the other response buttons. The size of each button is approximately $1 \times 1 \mathrm{~cm}$. Details of the technical implementation for this keyboard are described by Lacouture $(1995,1997)$. The keyboard was placed so that the participants were able to use their dominant hands. In the identification conditions, each response button corresponded to one of the possible stimuli and the button arrangement corresponded to the natural ordering of the stimuli from the smallest (leftmost key) to the largest (rightmost key). Two responses were used in the categorization task. One response category, labeled "A," was associated with the leftmost key on the response keyboard, and the other response category, labeled "B," was associated with the rightmost key. Thus, in the categorization task only the two extreme keys on the response keyboard were used for collecting responses. RT - the time elapsed between the onset of the stimulus on the screen and response keypress - was recorded and timed in milliseconds using MEL's timing routines.

In the vocal response conditions, a microphone and a voice key were used to collect the responses. For the identification task, the participants were required to say the numeral (1-10) that they considered correct for the current stimulus. For the (binary) categorization task, the participants were required to say the letter (A or B) that they considered correct for the current stimulus. A set of Coulborn modules relayed the voice key signal to the parallel port of the computer, allowing measurement of RT using MEL's timing routine. RT was measured as the elapsed time between the onset of the stimulus on the screen and the detection of a vocal response. Although RT was measured using voice key detection, the actual response made by the participant was manually entered on a computer keyboard by an experimenter sitting outside the experimental room. The experimenter listened to the participants' responses using a headset and monitored the presentation of the stimuli on a computer screen that duplicated the presentation of the stimuli to the participants. The experimenter noted whenever the voice key was accidentally triggered, and such trials were not analyzed; fewer than $1 \%$ of the trials were discarded for this reason.

The participant initiated each trial by pressing the START button. One hundred milliseconds later, one randomly selected stimulus was shown on the screen. The participant had to identify (or categorize) the stimulus either by pressing one of the appropriate response buttons (in the manual response conditions) or by naming the stimulus out loud (in the verbal response conditions). After onset, the stimulus remained on the screen until the participant provided a response.

One second after the participant's response had been recorded, feedback was provided for $1 \mathrm{sec}$ either in the form of a number corresponding to the ordinal position of the stimulus (identification task) or in the form of the corresponding correct Response Category A or B (categorization task). If the participant made an incorrect response, a low-frequency $(500-\mathrm{Hz})$ tone was generated for $500 \mathrm{msec}$. A trial ended with the presentation of a blank screen. If the participant waited more than $30 \mathrm{sec}$ before pressing the START button to begin the next trial, a short sequence of three tones was generated to regain the participant's attention.
Design and Stimuli. Each participant completed two sessions for each of the four conditions, for a total of eight experimental sessions over a 2-day period. In all conditions, the stimuli were the same 10 line segments individually presented horizontally in the center of the screen. The line segments were three pixels thick and appeared in white on a black background. From the participant's viewpoint, the stimuli appeared to be continuous lines. The length of each segment was $92,106,120,138,160,184,212,242,278$, or 320 pixel units (screen dots), and therefore the difference in length of adjacent stimuli was $15 \%$, to within screen resolution. When Weber's law holds, which is the case with line length in the range we study, stimuli spaced logarithmically are likely to be equally discriminable and, therefore, equally spaced along the decision axis. With their spacing well above Weber's fraction for line length $(2.9 \%$ as reported in Teghtsoonian, 1971), the stimuli should also be perfectly discriminable. In the identification tasks, the stimuli were given "correct" response labels of " 1 " to " 10 " according to increasing length. In the categorization task, adjacent stimuli were associated with different responses, with Stimuli 1, 3, 5, 7, and 9 associated with Response A and Stimuli 2, 4, 6, 8, and 10 associated with Response B.

The order of presentation of the four experimental conditions was counterbalanced across participants. Each session involved a total of 400 trials (40 for each stimulus) and lasted approximately $40 \mathrm{~min}$. The participants were instructed to respond as fast and accurately as possible.

\section{Results}

The data were averaged across participants for each stimulus. Trials associated with extreme RT values $(2 \%$ at each extreme of the percentile distribution for each condition) were removed. PC and mean correct RT were computed for each experimental condition - that is, only trials associated with correct responses were used in the RT analysis. ${ }^{6}$ The usual descriptive statistics are reported in Table 2. Within-subjects analyses of variance using a $2 \times 2$ design were performed for PC and mean RT using two factors: task (identification vs. categorization) and response mode (manual vs. vocal). No significant main or interaction effects were found for either factor.

In Figure 2, mean RT is plotted against stimulus position in each experimental condition. To make the results easier to understand, the figure shows four panels, each reporting the results of two experimental conditions. This allows one, on the one hand, to compare the results of the identification conditions with the results of the categorization conditions and, on the other hand, to compare the results of the manual conditions with the results of the vocal conditions. The results for each experimental condition appear in two of the four panels. As can be seen, the results are very similar in each condition when one compares the vocal condition with the manual con-

Table 2

Results of Experiment 1: Mean (With Standard Deviation) of Response Times (in Milliseconds) and Probability Correct (PC) for Each Experimental Condition

\begin{tabular}{lccc}
\hline \multirow{2}{*}{\multicolumn{1}{c}{ Condition }} & \multicolumn{2}{c}{ Response Time } & \\
\cline { 2 - 3 } & $M$ & $S D$ & PC \\
\hline Vocal identification & 1,308 & 447 & .72 \\
Manual identification & 1,268 & 409 & .67 \\
Vocal categorization & 1,395 & 524 & .70 \\
Manual categorization & 1,267 & 548 & .67 \\
\hline
\end{tabular}


(A)

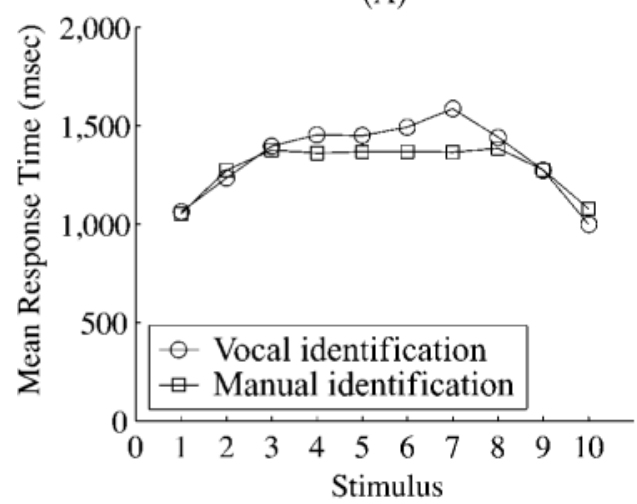

(C)

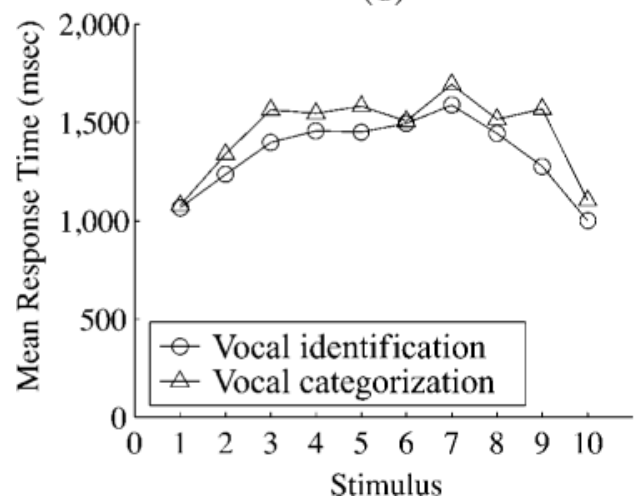

(B)

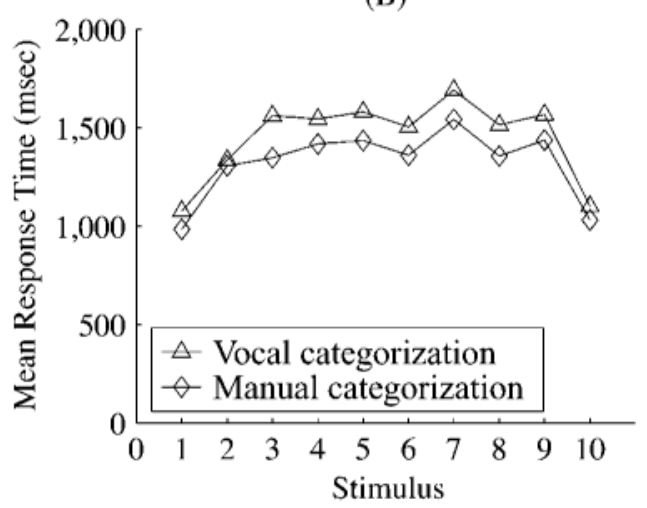

(D)

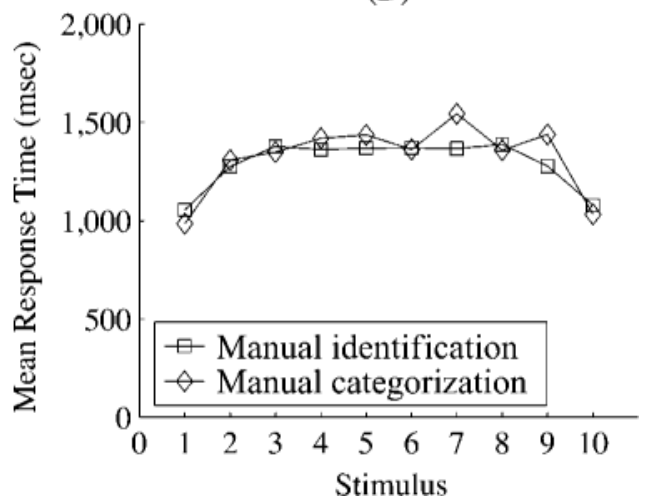

Figure 2. Results of Experiment 1: mean response time plotted against stimulus position. Each panel presents the result of two experimental conditions.

dition (panels A and B) or the identification task with the categorization task (panels $\mathrm{C}$ and $\mathrm{D}$ ). Note that mean RTs are shorter toward the ends of the stimulus range in all four conditions.

With the same organization as that of Figure 2, Figure 3 presents average PC plotted according to stimulus position for each experimental condition. As can be seen, $\mathrm{PC}$ tends to be higher toward the ends of the stimulus range. Taken together, Figures 2 and 3 show very small differences between the four conditions. Despite some variability in the pattern, the bow effect is clearly present in that the probabilities of correct response are higher and the RTs are (on average) shorter for stimuli located toward the ends of the stimulus range (see Lacouture, 1997, for a review of related data).

\section{EXPERIMENT 2}

Experiment 1 demonstrated that the overall pattern of RTs and probability of error are extremely similar for both the identification and categorization tasks using either vocal or manual responses. The same procedure and experimental design was used in Experiment 2, the difference being that only 1 participant completed 10 experimental sessions in each condition. This larger data set allows us to study the full distributions of RTs.

\section{Method}

Participant. One female graduate student who reported having normal vision and no motor handicap served as a participant.

Apparatus and General Procedure. The experiment was conducted with the same apparatus and general procedure used for Experiment 1 . The participant performed identification and categorization of the same visual stimuli under the same two response conditions (vocal and manual). The participant performed 10 sessions for each of the four experimental conditions (manual identification, vocal identification, manual categorization, and vocal categorization) for a total of 40 sessions. As in Experiment 1, there were 400 trials in each session. The participant first performed one session in each condition in the following order: manual identification, vocal identification, manual categorization, and vocal categorization. She then started over for a new round of four sessions following the same order. Ten rounds were performed over a 6-week period. No more than two sessions were run on the same day.

Design and Stimuli. The experimental design was, as in Experiment 1 , a $2 \times 2$ factorial design with the factors of task (identification vs. categorization) and type of responses (manual vs. vocal). The stimulus set was composed of the same 10 line segments used in Experiment 1, individually presented horizontally in the middle of the computer screen.

\section{Results}

The data were averaged for each stimulus in each condition. Trials associated with extreme RT values $(2 \%$ at each extreme of the percentile distribution for each condition) were removed. PC and mean correct RT were 
(A)

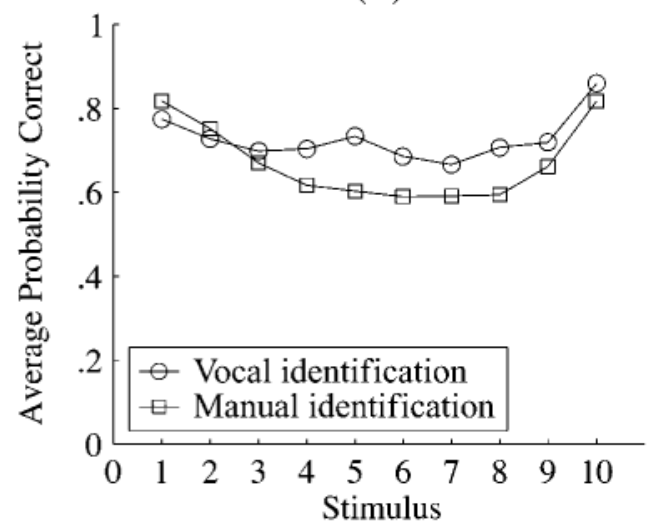

(C)

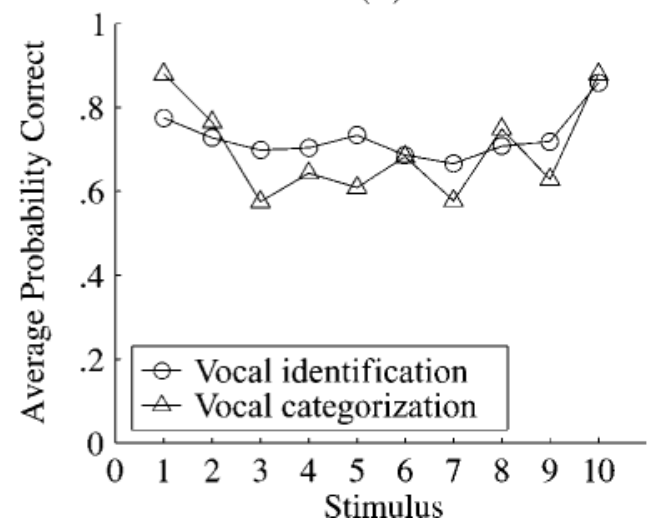

(B)

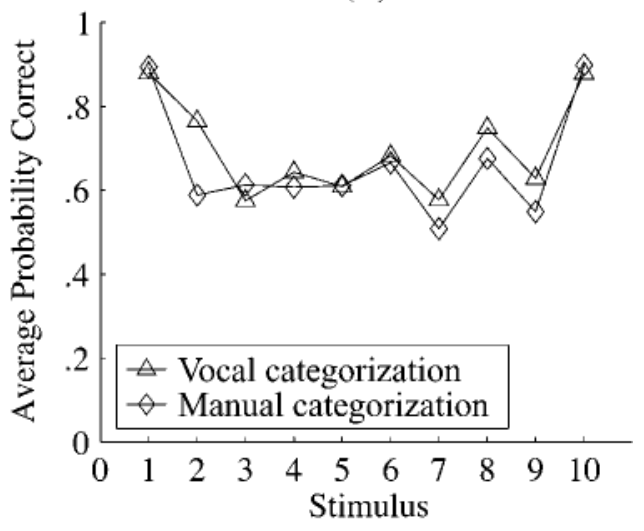

(D)

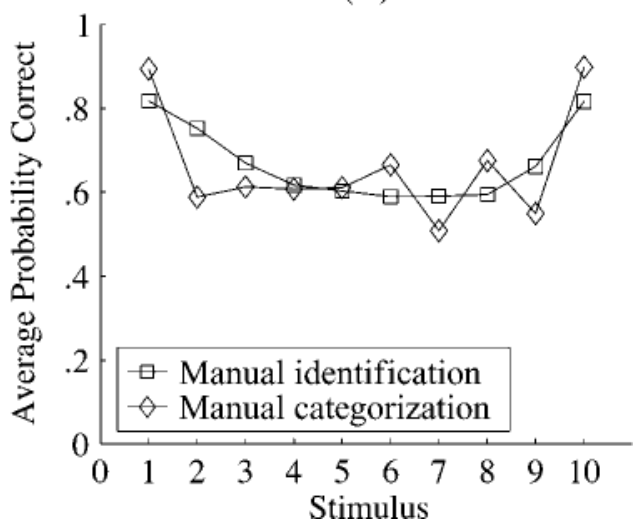

Figure 3. Results of Experiment 1: average probability correct plotted against stimulus position. Each panel presents the result of two experimental conditions.

computed for each of the experimental conditions. The usual descriptive statistics are reported in Table 3.

Visual inspection of Table 3 shows that the results closely resemble those obtained in Experiment 1. Vocal responses take, on average, slightly more time than manual responses and have slightly higher overall PCs. In Figure 4, mean RT plotted against stimulus position in each condition is reported. Again, the figure has four panels, each showing a comparison of the results in two of the four experimental conditions. Figure 5 shows average PC plotted against stimulus position in each condition. Each of the four panels of the figure allows for the comparison of two conditions. The results show similar patterns in each condition, with higher PCs and shorter RTs toward the ends of the stimulus range.

Analysis of RT distributions. The distribution of correct RTs for each stimulus in each condition was analyzed using maximum likelihood estimations. The best-fitting parameters were found for the gamma, ex-Gaussian, Gaussian, and Weibull functions. Using the AIC criterion, a superior fit to the empirical distributions was found in all cases using the ex-Gaussian function.

The ex-Gaussian function is the convolution of a Gaussian (normal) function with an exponential function. McGill (1963) first proposed the ex-Gaussian as a decision time model in cognitive processes. Mathematically, the ex-Gaussian probability density function $f$ is written as:

$$
f(t)=\frac{1}{\tau} \exp \left(\frac{\mu}{\tau}+\frac{\sigma^{2}}{2 \tau^{2}}-\frac{t}{\tau}\right) \Phi\left(\frac{t-\mu-\frac{\sigma^{2}}{\tau}}{\sigma}\right), t>0 .
$$

In this equation, the exponential function (exp) is multiplied by the value of the cumulative density function of the Gaussian function $(\Phi)$. The resulting ex-Gaussian function has three parameters: $\mu, \sigma$, and $\tau$. The first two parameters, $\mu$ and $\sigma$, correspond to the mean and $S D$ of

Table 3

Results of Experiment 2: Mean (With Standard Deviation) of Response Times (in Milliseconds) and Probability Correct (PC) for Each Experimental Condition

\begin{tabular}{lccc}
\hline \multirow{2}{*}{\multicolumn{1}{c}{ Condition }} & \multicolumn{2}{c}{ Response Time } & \\
\cline { 2 - 3 } & $M$ & $S D$ & PC \\
\hline Vocal identification & 1,181 & 379 & .90 \\
Manual identification & 1,047 & 188 & .84 \\
Vocal categorization & 1,354 & 495 & .87 \\
Manual categorization & 1,178 & 361 & .84 \\
\hline
\end{tabular}


(A)

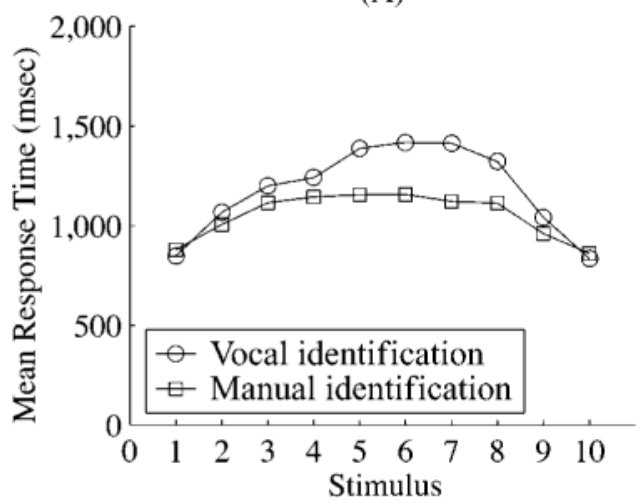

(C)

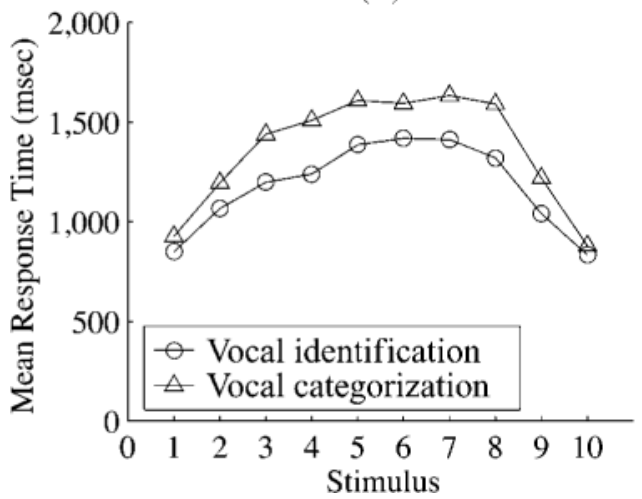

(B)

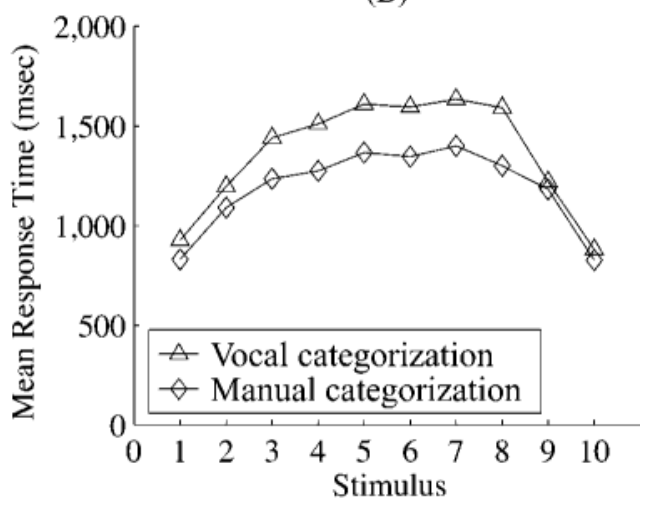

(D)

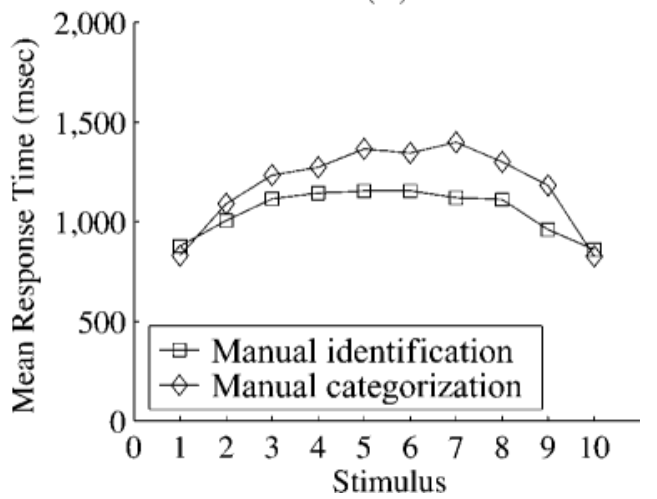

Figure 4. Results of Experiment 2: mean response time plotted against stimulus position. Each panel presents the result of two experimental conditions.

the Gaussian component, and the third parameter, $\tau$, is the mean of the exponential component.

In the framework of cognitive process RTs, this convolution can be seen as representing the overall distribution of two additive or sequential processes. As Luce (1986, chap. 6) proposed, the exponential process may be conceptualized as the decision component (i.e., the time required to decide which response to make) whereas the Gaussian component may be conceptualized as the transduction component (i.e., the sum of the time required by the sensory process and the time required to physically make the response). Although the theoretical proposition that cognitive process RTs are the sum of two additive processes is difficult to test, several researchers have

Table 4

Estimated Parameter Values of the Best-Fitting ex-Gaussian Function for the Reaction Time (in Milliseconds) Distribution of Correct Response for Each Stimulus in Each Condition

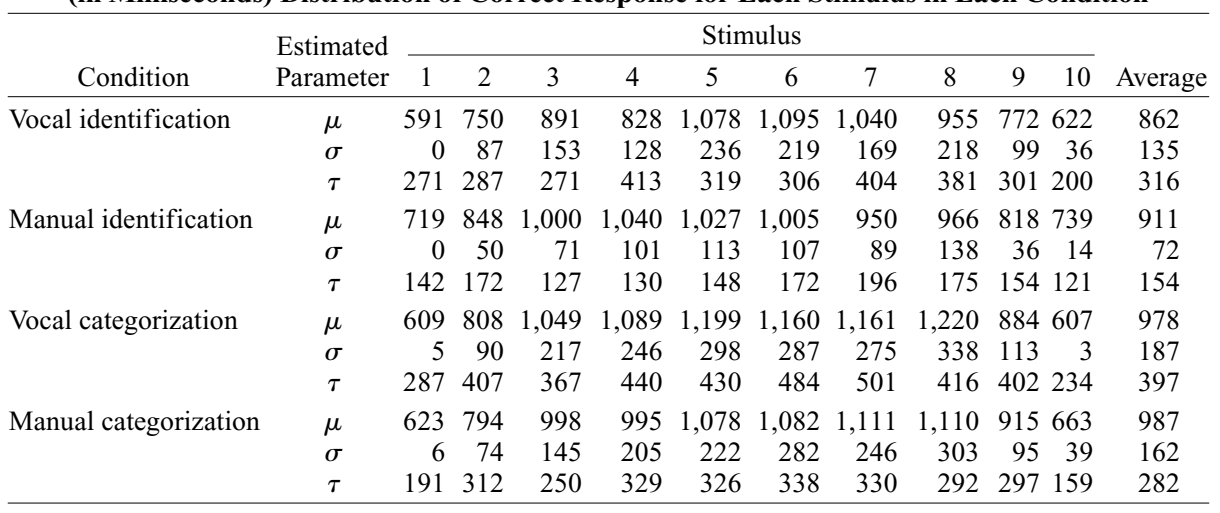


(A)

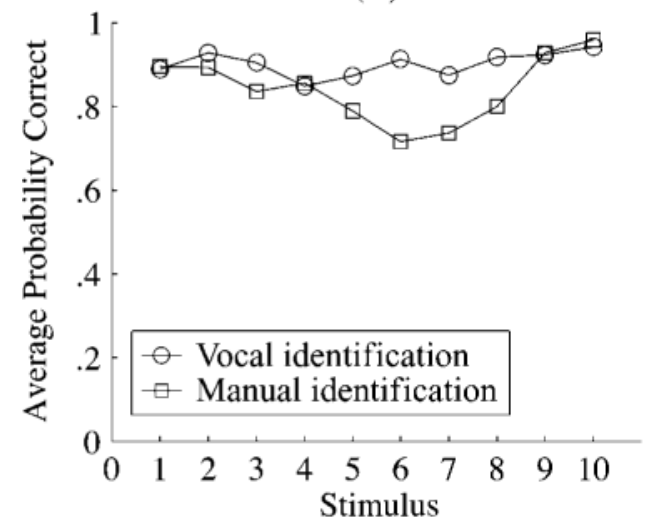

(C)

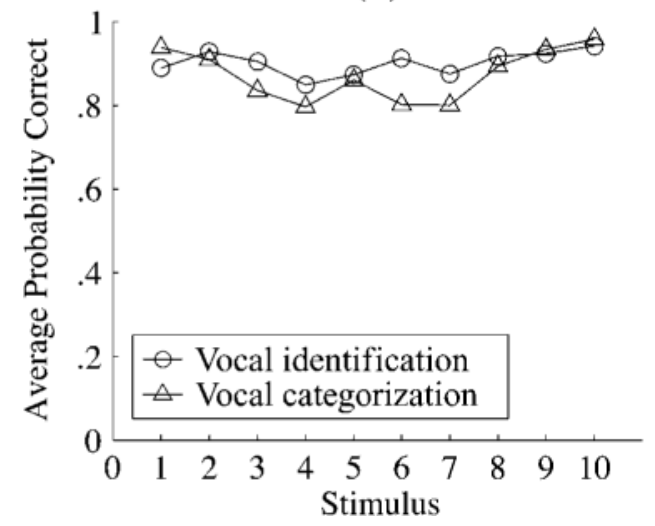

(B)

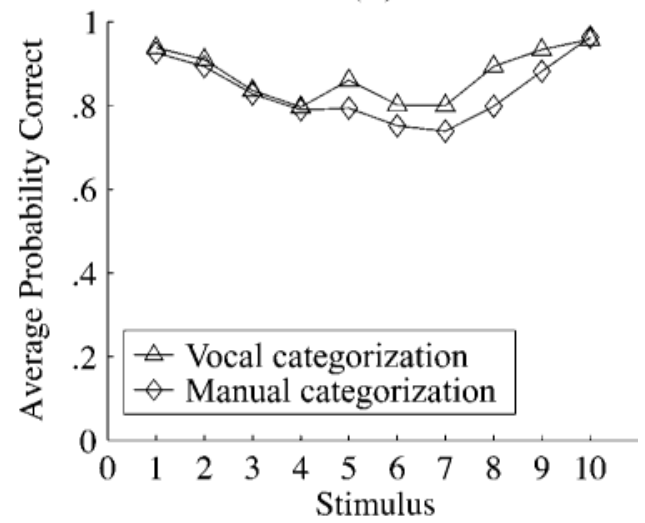

(D)

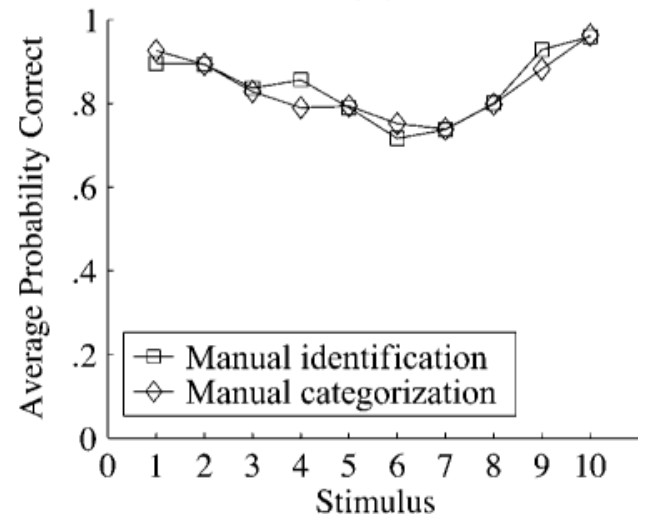

Figure 5. Results of Experiment 2: average probability correct plotted against stimulus position. Each panel presents the result of two experimental conditions.

demonstrated that the ex-Gaussian function provides a very good fit to several empirical RT distributions (Hockley, 1984; Hockley \& Corballis, 1982; Luce, 1986; Ratcliff \& Murdock, 1976). Other authors have suggested that, in the absence of any theoretical assumptions, the exGaussian function can be used effectively to characterize an arbitrary RT distribution (see Heathcote, Popiel, \& Mewhort, 1991). When the value of parameter $\tau$ tends toward zero, the shape of the ex-Gaussian function tends toward a normal function; when the parameter $\tau$ is larger than the parameter $\sigma$, the function is skewed toward the right and the length of the right tail is proportional to $\tau$; and when $\sigma$ tends toward zero, the ex-Gaussian function tends toward an exponential function.

Table 4 presents the estimated parameter values of $\mu, \sigma$, and $\tau$ for each stimulus in each condition and the overall average for each parameter in each condition. Figure 6 presents, for each stimulus in each condition, a histogram representing the distribution of RT with the overlaid best-fitting ex-Gaussian function. It can be seen that the empirical data are fit very well by the estimated functions. The results show that, in all cases, the distribution follows a skewed ex-Gaussian function. Toward the ends of the stimulus range, the parameter $\sigma$ of the ex-Gaussian function approaches zero and, thus, the distribution of RT tends toward an exponential function. Overall, categorization shows slightly larger parameter values. Also, the RT distribution of the vocal conditions appears to be characterized by a longer right tail (larger $\tau$ value). Note that there is no theoretical motivation for the distributions of RTs to be best fitted by the ex-Gaussian function; we use the fitted parameters as descriptive statistics and we do not attempt to interpret their significance.

\section{Simulating the Manual Absolute Identification Task}

In order to replicate the single-participant data obtained in Experiment 2, a simulation of the mapping model was run using the leaky competing accumulator decision process. As was described earlier and is shown in the top panel of Figure 1, the output from the mapping process was used as the input to the leaky competing decision process.

Given the close similarity between the manual and voice key data, we lose little in generality by choosing to fit the results of the manual identification condition. To do so, we adjusted two sets of parameters. On one hand, the level of noise at the input, hidden, and output levels 

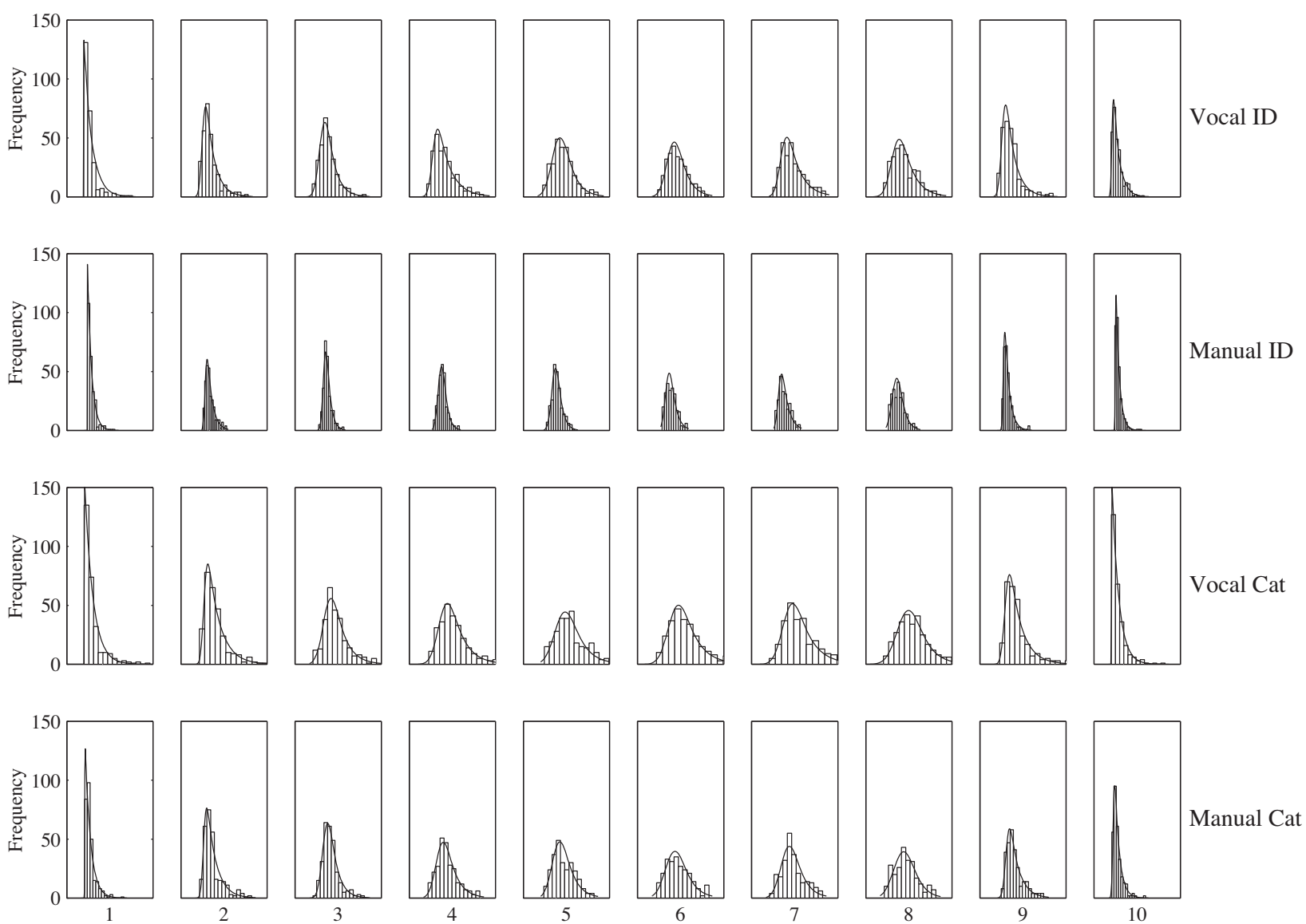

Stimulus

Figure 6. Response time distribution for each stimulus in each condition overlaid with the best-fitting ex-Gaussian function. ID, identification task; Cat, categorization task.

of the mapping process were manipulated by changing the $S D$ of the Gaussian random variable associated with each processing level-namely, $\gamma$ (input level), $\eta$ (hidden level), and $\varepsilon$ (output level). On the other hand, the parameters $l$ and $\beta$ of the leaky competing process were adjusted. As was mentioned earlier, the parameter $\Delta$, which controls the size of the simulation time steps, was set to 0.25 . We tried to fit jointly PC, mean RT, and $S D$ of RT. The parameter search was done by trial and error. Initially, we attempted to select the "best" parameter values using various criteria and searching algorithms. The noisy characteristics of the simulated process often led to nonconvergence of the search algorithms. Also, it appears difficult to derive an appropriate goodness-of-fit criterion for simultaneously fitting, say, the mean and variance of RTs and the PC. Therefore, we used a trialand-error approach with a systematic search of part of the parameter space. Van Zandt (2000) is an excellent contribution to related issues.

We obtain a good fit of the simulated data to the empirical data with the following parameters: input noise $\gamma=$ 0.035 , hidden noise $\eta=0.02$, output noise $\varepsilon=0.02$, and, for the competing accumulators parameters, $\beta=0.3$ and $l=0.2$. Although in the present article we report a new data set, the three parameters of the mapping solution are identical to those used in our previous work, and those for the variance of the noise at each level of the mapping process are close to the parameter values used previously.

When simulating the response process, we assumed that an overt response is made when a single unit remains active and has reached a stable level of activation. Because of the stochastic nature of the process, the activation level of the winning unit fluctuates around a fixed value. We consider the network to be stable when the change in activation for the single winning unit remains smaller than a fixed value $\gamma$ for 15 network steps. In the present simulation, we used the criterion $d x_{w}<\gamma=.01$. Using this criterion, we simulated 300 identification trials for each of the 10 stimuli. Mean RT was computed for each stimulus using trials in which correct responses were made; results on error responses are summarized later. Note that the simulations do not involve any learning and the simulated results are taken to correspond to the asymptotic performance. Note also that performance in absolute identifica- 

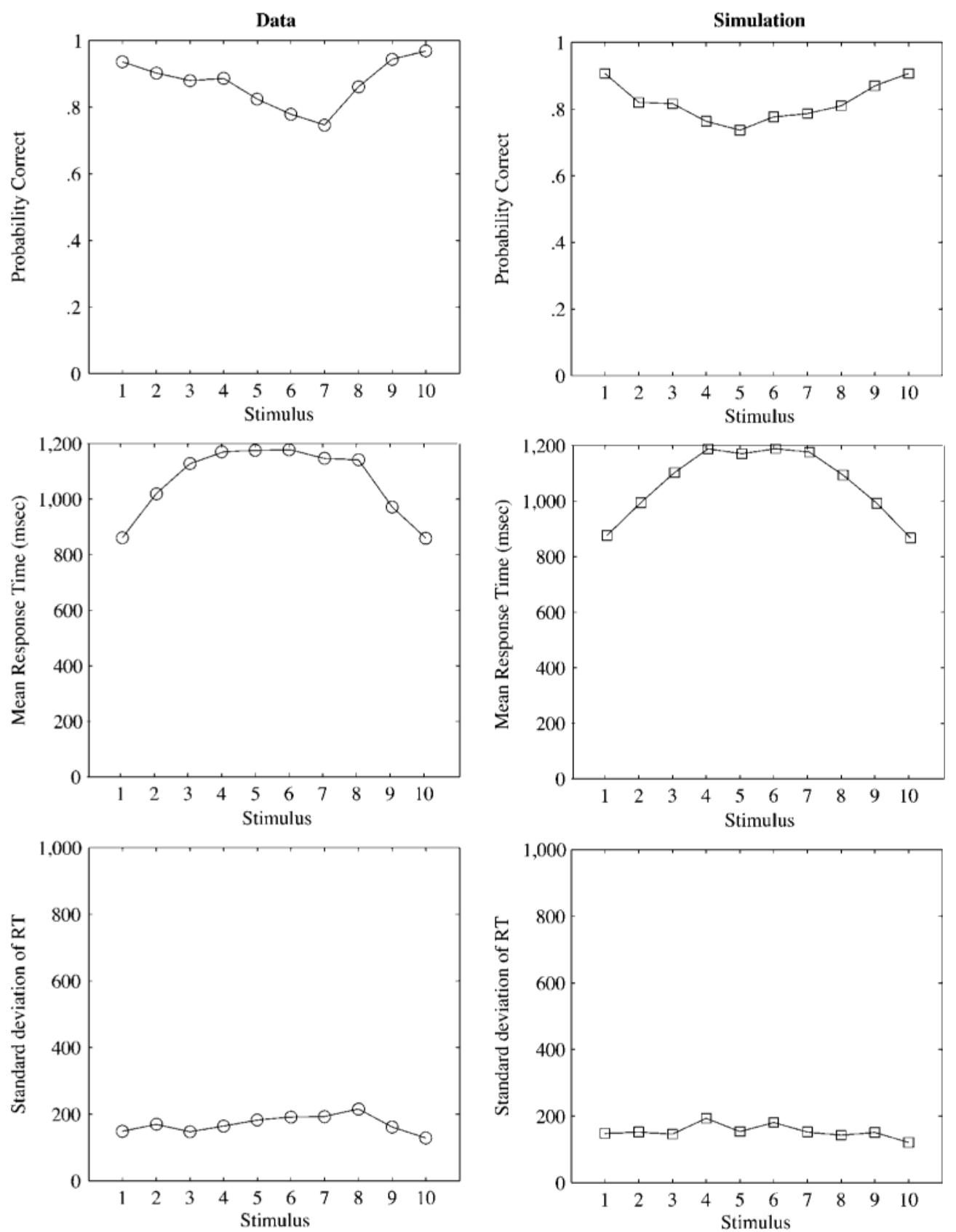

Figure 7. Data (left panels) and simulation (right panels) for the manual identification task. The top panels show probability correct, the middle panels show mean response time (RT), and the bottom panels show standard deviations of RTs. Results are plotted against stimulus position. The empirical data are from Experiment 2.

tion of unidimensional stimuli asymptotes quickly and shows only slight improvement with practice (see Shiffrin $\&$ Nosofsky, 1994, for a comment on that issue). A fascinating exception to this statement may be found in Rouder, Morey, Cowan, and Pfaltz (2004).

A linear regression was performed to establish the correspondence between the number of simulation cycles (steps) and real time. The resulting regression func- tion is $t=259.6+3.9 c$, where $c$ is the simulation time in network cycles and $t$ is the corresponding real time in milliseconds. The fit of the equation is very good, with $R^{2}=.96$. Figure 7 shows the relation between the empirical (left panels) and simulated results (right panels) when the regression function above is used to convert number of simulation steps to real time. Overall, the simulated process closely matches the data. 


\section{Confusion Matrices and RTs on Incorrect Trials}

Table 5 shows the whole confusion matrix for the data (top) and simulation (bottom). However, remember that the parameters of the simulated process were adjusted to simultaneously fit the average PC and the mean RT associated with each stimulus, not the whole confusion matrix; we proceeded in this way because of the relatively small proportions of errors. The two matrices have similar structures, and although no attempt was made to replicate the whole data confusion matrix using the simulated model, the correspondence between it and the simulation results is very good. For instance, in both matrices errors associated with stimulus $i$ typically involve response $i+1$ or $i-1$, with very low probabilities of response $i+2, i-2$, or others. The $R^{2}$ value between the complete matrices is .99 , and the $R^{2}$ value after the main diagonal (PC) in each matrix is removed is .82, which indicates a very good fit between the probabilities of errors for the data and the simulation.

Table 6 reports the results of the comparison of mean RTs for correct and incorrect responses from the data and from the simulation. In both cases, the mean RTs associated with incorrect trials are longer than the mean RTs associated with correct trials, and there is a good match despite a slightly larger difference between the percentages of correct and error responses for the simulated results. Again, remember that when the parameters of the model were adjusted, no attempt was made to fit the error data, mainly because of the relatively small percentages of such errors.

\section{Response Time Distributions of the Simulated Process}

The network does not exhibit perfect performances when the leaky competing decision process is noisy, and the RTs vary for a given stimulus from trial to trial. A good model of the task should reproduce not only PC, mean RT, and $S D$ of RT, but also the overall shape of the RT distributions.

As was mentioned earlier, we simulated 300 identification trials for each stimulus, for a total of 3,000 trials. This allows us to obtain a good representation of the RT distributions. Using "correct" trials, best-fitting parameters for the gamma, Gaussian, ex-Gaussian, and Weibull functions were estimated for each of the 10 simulated distributions (one for each of the stimuli). The ex-Gaussian function gave the best fit in every case. Figure 8 presents both the empirical distributions (from Experiment 2) and the simulated distributions for each stimulus in the manual identification task overlaid with the best-fitting exGaussian function. For both the empirical and simulated data, the distributions are well represented by such exGaussian functions. Also in both cases, the shape of the distributions approaches an exponential function toward the ends of the stimulus range (parameter $\sigma$ tends toward zero). In Figure 9, quantile-quantile (QQ) plots are reported which illustrate, for each stimulus, the correspondence between the empirical and the simulated RT distributions. The QQ graphs plot the quantile values for the empirical distribution ( $x$-axis) against the quantile values for the simulated distribution ( $y$-axis), where di-

Table 5

Confusion Matrix for the Data and Simulation

\begin{tabular}{|c|c|c|c|c|c|c|c|c|c|c|}
\hline \multirow[b]{2}{*}{ Stimulus } & \multicolumn{10}{|c|}{ Response } \\
\hline & 1 & 2 & 3 & 4 & 5 & 6 & 7 & 8 & 9 & 10 \\
\hline & \multicolumn{10}{|c|}{ Data } \\
\hline 1 & 89.6 & 10.4 & 0 & 0 & 0 & 0 & 0 & 0 & 0 & 0 \\
\hline 2 & 4.1 & 89.3 & 6.6 & 0 & 0 & 0 & 0 & 0 & 0 & 0 \\
\hline 3 & 0 & 8.5 & 83.6 & 7.9 & 0 & 0 & 0 & 0 & 0 & 0 \\
\hline 4 & 0 & 0 & 6.9 & 85.6 & 6.4 & 1.1 & 0 & 0 & 0 & 0 \\
\hline 5 & 0 & 0 & 0 & 10.9 & 78.9 & 9.1 & 1.1 & 0 & 0 & 0 \\
\hline 6 & 0 & 0 & 0 & .3 & 18.0 & 71.6 & 9.8 & .3 & 0 & 0 \\
\hline 7 & 0 & 0 & 0 & 0 & .5 & 15.1 & 73.7 & 10.5 & .3 & 0 \\
\hline 8 & 0 & 0 & 0 & 0 & 0 & .8 & 13.9 & 80.0 & 5.3 & 0 \\
\hline 9 & 0 & 0 & 0 & 0 & 0 & 0 & .3 & 3.8 & 92.8 & 3.1 \\
\hline \multirow[t]{2}{*}{10} & 0 & 0 & 0 & 0 & 0 & 0 & 0 & 0 & 4.1 & 95.9 \\
\hline & \multicolumn{10}{|c|}{ Simulation } \\
\hline 1 & 90.7 & 9.3 & 0 & 0 & 0 & 0 & 0 & 0 & 0 & 0 \\
\hline 2 & 8.3 & 82.0 & 9.7 & 0 & 0 & 0 & 0 & 0 & 0 & 0 \\
\hline 3 & 0 & 9.0 & 81.7 & 9.3 & 0 & 0 & 0 & 0 & 0 & 0 \\
\hline 4 & 0 & 0 & 9.0 & 76.3 & 14.7 & 0 & 0 & 0 & 0 & 0 \\
\hline 5 & 0 & 0 & 0 & 16.7 & 73.7 & 9.7 & 0 & 0 & 0 & 0 \\
\hline 6 & 0 & 0 & 0 & .3 & 9.7 & 77.7 & 12.3 & 0 & 0 & 0 \\
\hline 7 & 0 & 0 & 0 & 0 & 0 & 14.0 & 78.7 & 7.3 & 0 & 0 \\
\hline 8 & 0 & 0 & 0 & 0 & 0 & .7 & 11.3 & 81.0 & 7.0 & 0 \\
\hline 9 & 0 & 0 & 0 & 0 & 0 & 0 & 0 & 7.7 & 87.0 & 5.3 \\
\hline 10 & 0 & 0 & 0 & 0 & 0 & 0 & 0 & 0 & 9.3 & 90.7 \\
\hline
\end{tabular}

Note-Each entry in the matrix gives the probability of the given response for the given stimulus. 
Table 6

Mean (With Standard Deviation) of Response Times (in Milliseconds) for Correct and Incorrect Responses for the Data and the Simulation

\begin{tabular}{lccccc}
\hline \multirow{2}{*}{ Trials } & \multicolumn{2}{c}{ Data } & & \multicolumn{2}{c}{ Simulation } \\
\cline { 2 - 3 } \cline { 6 - 7 } \multicolumn{1}{c}{ Trials with correct responses } & $M$ & $S D$ & & $M$ & $S D$ \\
\hline Trials with incorrect responses & 1,047 & 181 & & 1,057 & 181 \\
\hline
\end{tabular}

agonal lines in those plots correspond to a perfect fit of the simulation to the data. The figure shows, for all stimuli, a very good correspondence between the empirical and the simulated distributions.

\section{Simulating the Manual Categorization Task}

The bottom panel of Figure 1 shows a schematic view of the proposed model for simulating the categorization task described in this article. This architecture assumes that the categorization process involves an extra processing layer with two units connected to the identification decision model. Two decision units are used to implement the categorization process, with one unit corresponding to Category A and the other to Category B. Response "A" should be made when one of Stimuli 1, 3, 5, 7, and 9 is presented, whereas response "B" should be made when one of Stimuli 2, 4, 6, 8, and 10 is presented. The categorization process is implemented by connecting Units $1,3,5,7$, and 9 of the identification layer to Response Unit A and connecting Units 2, 4, 6, 8, 10 of the identification layer to Response Unit B. As in the identification layer, the two categorization units implement a leaky competing accumulator process and the processing in the categorization module is done simultaneously with the identification process. For simplicity, we assume that each accumulator in the identification module is connected to the correct accumulator of the categorization module by a direct connection (of weight 1 ). Thus, as activation grows in the identification units, it propagates directly to the categorization units. Since the categorization layer implements a leaky competing accumulator process, we used the same stability criterion and assumed that an overt response is made when activation, for the winning accumulator, remains stable for 15 network time steps. Note that one accumulator from the identification layer will also win its race (at the identification level), but we assume that it does not trigger an overt response.

The dynamics of the simulation show that the activation for the winning unit in the categorization module stabilizes after the activation for the winning unit in the identification module has stabilized. Although the group data of Experiment 1 had no significant differences across conditions, the individual data of Experiment 2 yielded small but noticeable differences between the manual identification and manual categorization conditions (see Figures 4 and 5). We first simulated the categorization process using, in the categorization layer, the same values of leakage and inhibition parameters $(l$ and $\beta)$ as in the identification layer, but the results produced much longer RTs for the categorization process than for the identification process. Thus, the amount of leakage for the categorization units was adjusted to replicate results from Experiment 2.

Leakage and inhibition parameters in the identification module were set to $l=0.2$ and $\beta=0.3$, respectively (as for the simulation of the identification task), and in the categorization module they were set to $l=0.6$ and $\beta=0.3$, respectively. Figure 10 shows mean RT (top panels) and average PC (bottom panels) plotted against stimulus position for the data of Experiment 2 (left pan-

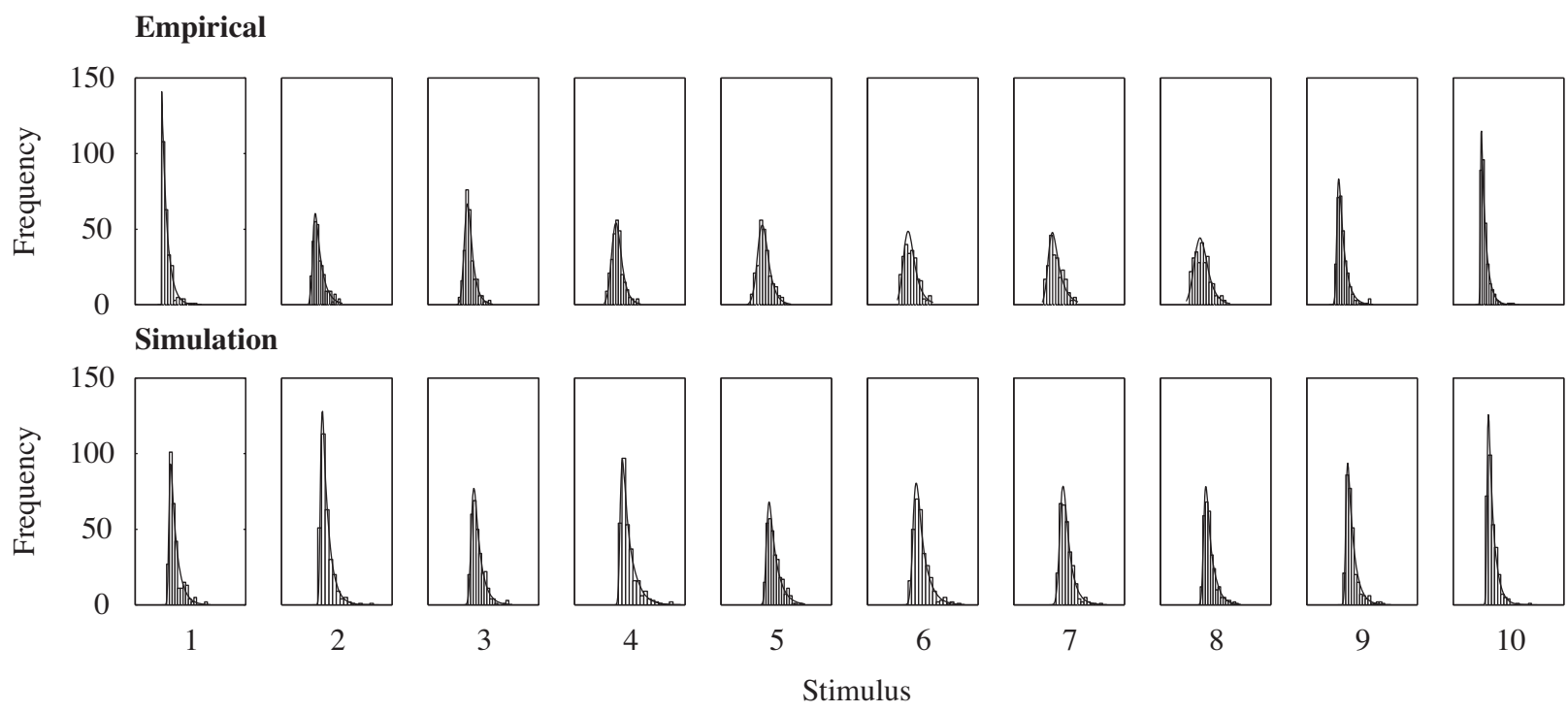

Figure 8. Response time distribution overlaid with the best-fitting ex-Gaussian function for each stimulus in the manual identification condition. The top panels show the empirical results from Experiment 2, and the bottom panels report the simulation results. 


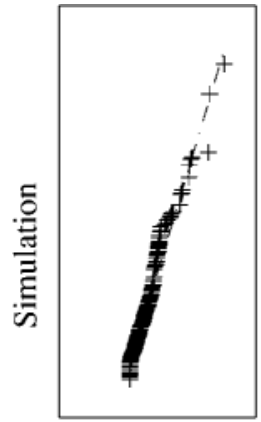

1

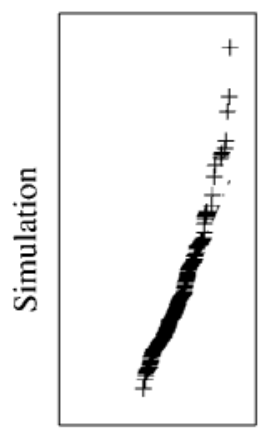

6

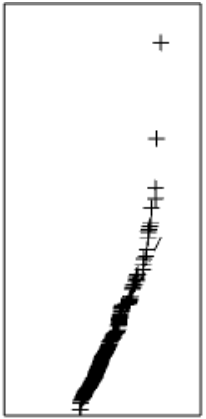

2

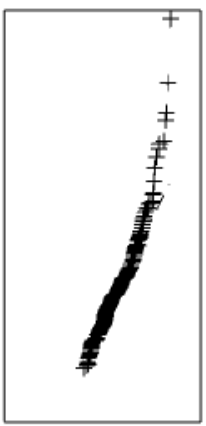

7

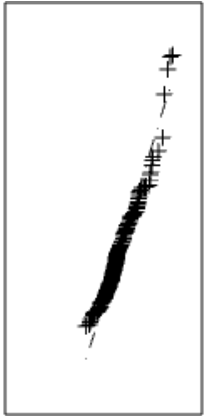

3

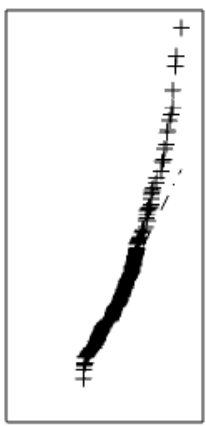

8

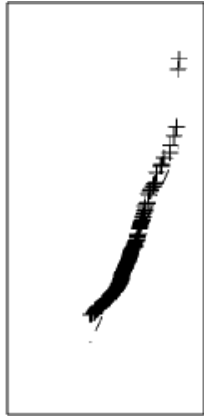

4

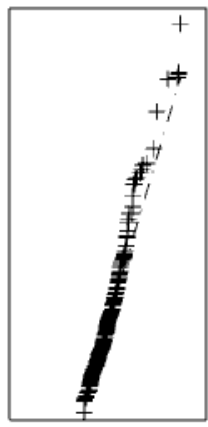

9

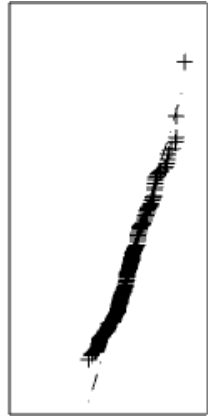

5

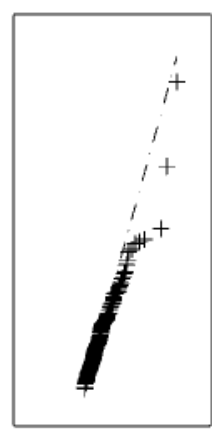

10

Data

Figure 9. Quantile-quantile plots of data ( $x$-axis) against simulation results ( $y$-axis) for the response time distribution for each stimulus in the manual identification task.

els) and the simulation (right panels). The model reproduces the empirical data very well. Note that RTs for Stimuli 1 and 10 are practically the same in both categorization and identification tasks, whereas the RTs for the intermediary stimuli gradually become longer in the categorization task than in the identification task. In a first implementation of the model for the categorization task, we assumed that the identification and categorization processes were carried out sequentially - that is, the network completed the identification process (there is only one stable unit at the identification layer) before allowing the activation to propagate to the categorization layer. This implementation produced, for each stimulus, categorization times that were, on average, longer than identification times by a constant value, which is not the case for the data. Allowing the activation to propagate directly from the identification layer to the categorization layer and having both decision processes run simultaneously allowed the model to produce similar RTs for Stimuli 1 and 10 in both the categorization and identification tasks, and longer RTs for intermediate stimuli in the categorization task, in agreement with the data. A similar implementation of the propagation of activation in a neural network, called cascade propagation, was proposed by McClelland (1979).

We also analyzed the RT distributions given for each stimulus by the simulation of the categorization task and found results that are very similar to what was found for the identification task. In all cases, the distribution was best represented by the ex-Gaussian function and there was a very good match between the simulated and empirical results. We do not report the details of those analyses, since they appear to be redundant with the results reported for the identification task.

\section{SUMMARY AND FUTURE DIRECTIONS}

The empirical results presented in this article reproduce several of the classical phenomena found in unidimensional absolute identification. The factorial design showed that the bow effect occurs for both manual and vocal responses when the participants are required to absolutely identify 10 line lengths. The overall results show no significant effect of the response modality on RT or PC. This result is congruent with those of Lacouture and Lacerte (1997), and, together, they suggest that the bow effect cannot be explained by simple "motor" factors or response "biases."

The empirical results for PC are extremely similar for the (unidimensional) absolute identification task and for the particular (unidimensional) binary categorization task presented here. The data of Experiment 1 showed no significant differences in RT or PC when these identification and categorization tasks were compared. We 
Data
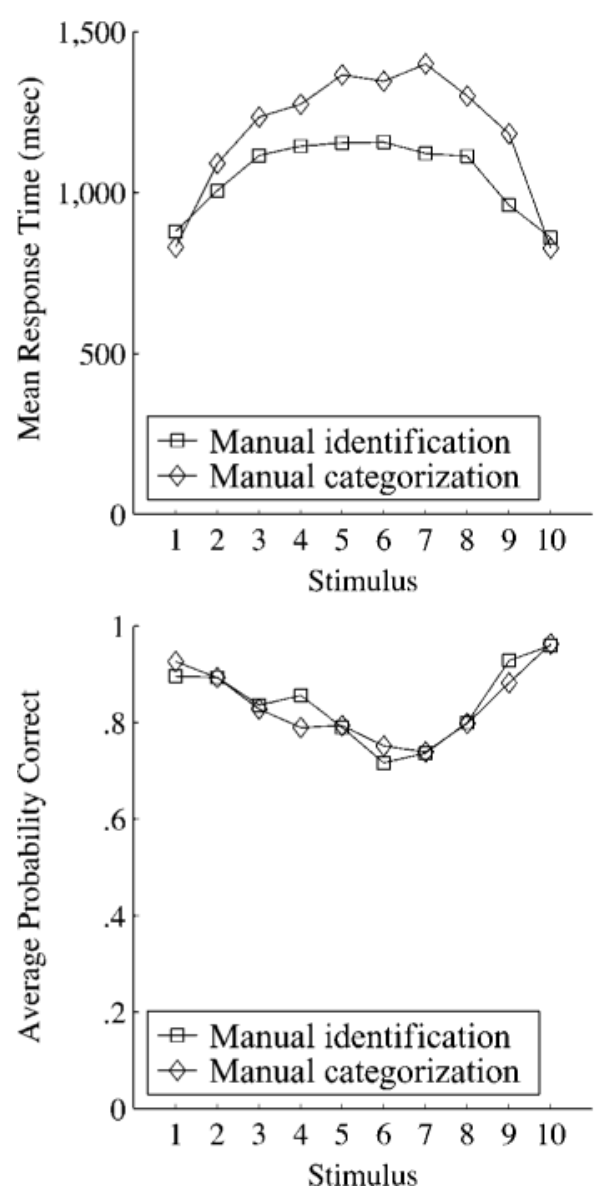

Simulation
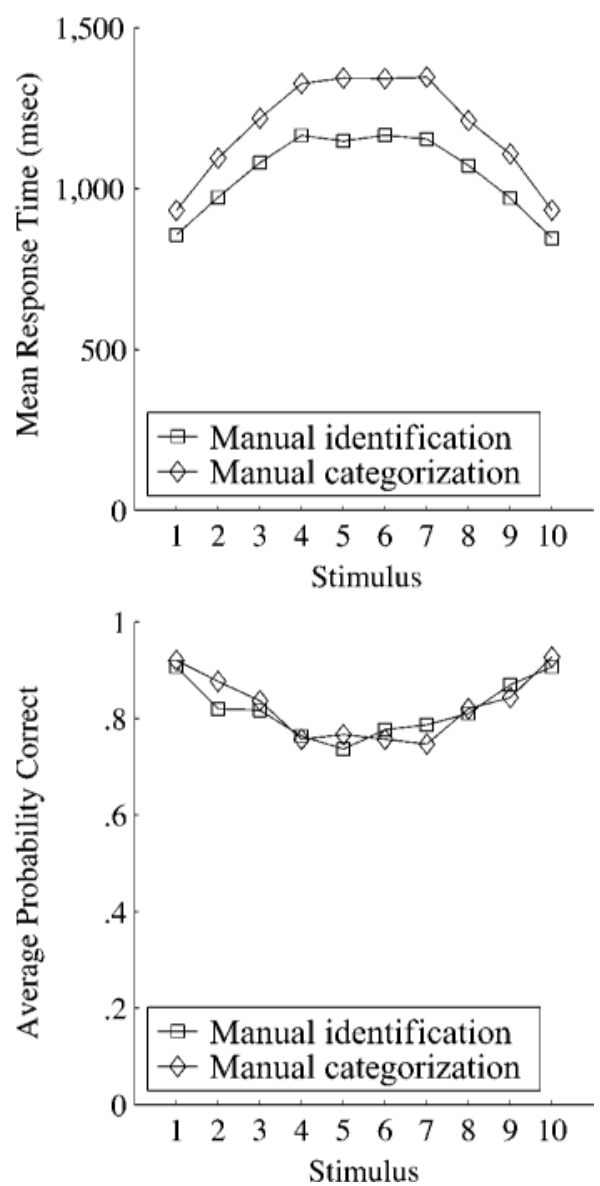

Figure 10. Mean response time and probability correct plotted against stimulus position for the manual identification and manual categorization tasks. The left panels present the data from Experiment 2, and the right panels show the simulation results.

fit the latter results by assuming an additional processing stage in which the contents of each of the accumulators associated with the identification responses " 1 " to " 10 " are mapped in the required manner to one of the two (response) categories. This process is congruent with the individual participant data of Experiment 2, in which slightly longer RTs are observed in the categorization task. Lacouture et al. (1998) have already shown that the same type of decision process can be used to model identification and categorization of unidimensional stimuli when adjacent stimuli (responses) are grouped to form categories.

The overall empirical and theoretical results relating the unidimensional identification data with those of the special unidimensional categorization task are very interesting, since they encourage us to explore the possibility that a similar categorization task could become a useful empirical and theoretical alternative to absolute identification tasks when the stimuli are multidimensional. As we discussed earlier in this article, it is very difficult to obtain sensible measures of RT in the identi- fication of multidimensional stimuli, and the categorization task circumvents this issue. We have preliminary data on such a multidimensional task that suggest that this approach is viable. Also, we have developed a multidimensional version of the (unidimensional) mapping solution presented in Lacouture and Marley (1995) and have begun to develop versions of our model for multidimensional absolute identification and categorization tasks. In these extensions of the model, as in the unidimensional version presented here, the categorization task is "naturally" built on top of the identification task, which suggests both a mechanism by which the task might be performed and the possibility that the task may become increasingly difficult for participants as the dimension increases - although, as the data of this article show, the participants had little difficulty with the unidimensional version of the special categorization task.

As was summarized above, our model of the categorization process assumes that as activation grows in the identification units, it propagates directly to the categorization units. This processing structure has some simi- 
larity to that of the exemplar-based approach to categorization, extensively developed by Nosofsky and his collaborators (see, e.g., Nosofsky \& Palmeri, 1997), one difference being that our mapping process gives an alternative, and perhaps more detailed, representation of the mapping from the external stimuli to the internal (exemplar-based) representation. A second approach would use a series of hyperplanes to divide the (2-D) hidden unit representation into distinct regions, each of which is then associated with a unique categorization response. This alternative processing architecture is similar to that of general recognition theory (GRT), extensively developed by Ashby and his collaborators (see, e.g., Ashby, 2000; Ashby \& Maddox, 1993). Both the exemplar-based and the GRT approaches provide excellent fits to a large amount of categorization (and a smaller amount of identification) data, and there is considerable discussion in the literature of the merits of each approach (see the references above). However, to our knowledge there is no data other than our own for a categorization task with the complex structure that we discuss in this article, which we believe can yield important additional tests of these and other models (Alfonso-Reese, Ashby, \& Brainard, 2002, do discuss complexity issues). Recent extensions of these approaches add more detail regarding sensory representations (see, e.g., Cohen \& Nosofsky, 2003, and the related Lamberts, 2000). As we obtain further data using our special categorization task, especially for multidimensional stimuli, we will compare these various theoretical approaches, thus generating important new links between identification and categorization, incorporating the sensory and cognitive aspects of both tasks.

The overall pattern of results of Experiment 1 was reproduced in Experiment 2, in which a single participant performed 10 sessions for each of the four experimental conditions. This larger data set allowed us to fit the full distribution of RTs for each stimulus. Among the various theoretical distributions studied, the ex-Gaussian gave the best fit in all cases.

We simulated the manual identification task with an extension of our mapping model, in which independent linear accumulators with thresholds were replaced with leaky competing integrators. The simulation gives excellent fits to the data. Not only did the simulation reproduce the observed mean and $S D$ of RTs and the overall PC, but it also produced a close match between the shape of the simulated and empirical RT distributions. As with the empirical data, the simulation produced RT distributions that were well represented by the ex-Gaussian function. Also, for both the data and the simulations, the RT distributions associated with stimuli toward the end of the range approach the exponential form. We do not propose any theoretical reasons as to why the simulated or empirical processes produce ex-Gaussian functions. Nonetheless, the correspondence between the empirical and simulated results (both being fit by ex-Gaussians) is clearly shown in the QQ plot of Figure 9.
The important issue of sequential effects has not been addressed thus far in this article (or in our prior work). This is partly due to a strategic choice on our part to concentrate first on other major effects, but, of course, to defend this strategic choice we must be able to revise and/or extend our model in a reasonable way to include such sequential effects. Performance in absolute identification is subject to strong sequential effects (see, e.g., Holland \& Lockhead, 1968; Lacouture, 1997; Lockhead, 1984; Mori, 1989; Ward \& Lockhead, 1970, 1971), with the response made on a given trial, and the time to make it, depending on the stimuli presented and responses made on previous trials. Several researchers have shown that sequential effects become larger as the task becomes more difficult.

Assimilation is the sequential dependency observed between the response at trial $n\left[\mathrm{R}_{N}^{(n)}\right]$ and the stimulus at trial $n-1\left[\mathrm{~S}_{N}^{(n-1)}\right]$, in which there is a tendency for participants to reduce the distance - as computed from the ordinal position-between $\mathrm{S}_{N}^{(n-1)}$ and $\mathrm{R}_{N}^{(n)}$. Contrasts are the sequential dependencies between $\mathrm{R}_{N}^{(n)}$ and $\mathrm{S}_{N}^{(n-2)}$, $\mathrm{S}_{N}^{(n-3)}$, and so forth; such dependencies on previous stimuli up to lag 8 have been reported (see, e.g., Ward \& Lockhead, 1970, 1971). Contrasts show up in the data as a tendency of participants to increase the distance - as computed from the ordinal position between $\mathrm{R}_{N}^{(n)}$ - and the stimuli prior to $\mathrm{S}_{N}^{(n-1)}$. We now briefly introduce three ways in which our current formulation might be revised to explain such effects. Considerable work is needed to develop and test these ideas, although each is similar to extant interpretations of sequential effects.

In the current implementation of the model, the (mean) psychological representation is bounded in $[0,1]$ through the computation of the difference ratio of Equation 1. The most natural way to begin the introduction of sequential effects, and one that is similar to other such proposals in the literature, is to replace this quantity by the quantity

$$
\left[\frac{\mathrm{S}_{N}^{(n)}(i)-\mathrm{S}_{N}^{(n-1)}(j)}{\mathrm{S}_{N}(\mathrm{U})-\mathrm{S}_{N}(\mathrm{~L})}\right],
$$

where $\mathrm{S}_{N}^{(n)}(i)$ and $\mathrm{S}_{N}^{(n-1)}(j)$ are the stimuli presented on trial $n$ and trial $n-1$, respectively. However, such a representation no longer immediately deals with end-anchor effects (which have not been considered in the literature in which the above type of representation has been proposed).

We now turn to the other two possible interpretations of sequential effects. Remember that two sources of noise affect the representation in Equation 2-namely, sensory noise and hidden unit noise. In each case, we assume that the noise follows a Gaussian distribution with mean zero and an estimated variance. Both sources of noise are added to the stimulus representation at each time step, and the model implements a mechanism whereby multiple noisy samples of the sensory input are taken within a trial. In Equation 3, superscript $k$ denotes the within-trial 
time steps. Let $r(n)$ be the number of time steps required at trial $n$ to produce an overt response. A memory trace for the psychological representation can be implemented in such a way that the internal representation at any given time step $k$ of trial $n$ is given by

$$
h_{N}^{k}=H_{N}^{k}+p_{1} h_{N}^{r(n-1)}+p_{2} h_{N}^{r(n-2)}+\ldots,
$$

where $H_{N}^{k}$ is the noisy internal representation at step $k$ within trial $n$, given by Equation 2, and $h_{N}^{r(n-1)}$ is the internal representation when the response was made at the end of trial $n-1$; similarly, $h_{N}^{r(n-2)}$ is the internal representation at the time that a response was made at the end of trial $n-2$, and so on. The coefficients $p_{1}, p_{2}, \ldots$ are "memory" parameters which, when positive, implement assimilation effects and, when negative, implement contrast effects. The resulting internal representation $h_{N}^{k}$ is, for trial $n$ and intratrial sample $k$, a noisy representation that is affected, on one hand, by sensory and decision noise and, on the other hand, by a memory trace from previous trials. This extension of our model corresponds to the incorporation of an autoregressive component similar to the memory mechanism proposed by Ward and Lockhead (1971) to model sequential effects.

Alternatively, or in addition to the above, we might assume that at the beginning of each trial the activation of each of the accumulators in the decision module is not reset to zero. Again, let $r(n)$ denote the number of time steps required to produce a response at trial $n$. Implementing a memory mechanism for each of the accumulators in the leaky competing decision process, we have, for accumulator $j$ at the beginning of trial $n$, the "residual" activation:

$$
\mathrm{A}_{N}^{0}(j)=q_{1} \mathrm{~A}_{N}^{r(n-1)}(j)+q_{2} \mathrm{~A}_{N}^{r(n-2)}(j)+\ldots,
$$

where $j=1,2,3, \ldots N$, and $\mathrm{A}_{N}^{r(n-k)}(j)$ is the activation in accumulator $j$ when the response was made at the end of trial $(n-k), k=1,2, \ldots$ The coefficients $q_{1}, q_{2}, \ldots$ are "memory" parameters that control the memory traces. Obviously, considerable further work is required to test the value of these ideas, as well as that of others, in using the mapping model with leaky competing integrators in the explanation of sequential effects in absolute identification and categorization. Nonetheless, the results reported in this article show that the mapping model with leaky competing integrators is an excellent framework for modeling the responses made, and the time to make them, in unidimensional absolute identification and categorization, and has considerable potential for extension and application to related multidimensional tasks.

\section{REFERENCES}

Alfonso-Reese, L. A., Ashby, F. G., \& Brainard, D. H. (2002). What makes a categorization task difficult? Perception \& Psychophysics, 64, 570-583.

AsHBY, F. G. (2000). A stochastic version of general recognition theory. Journal of Mathematical Psychology, 44, 310-329.
Ashby, F. G., \& LeE, W. W. (1991). Predicting similarity and categorization from identification. Journal of Experimental Psychology: General, 120, 150-172.

Ashby, F. G., Lee, W. W., \& Balakrishnan, J. D. (1992). Comparing the biased choice model and multidimensional decision bound models of identification. Mathematical Social Sciences, 23, 175-197.

Ashby, F. G., \& Maddox, W. T. (1993). Relations between prototype, exemplar, and decision bound models of categorization. Journal of Mathematical Psychology, 37, 372-400.

Berliner, J. E., \& Durlach, N. I. (1973). Intensity perception: IV. Resolution in roving-level discrimination. Journal of the Acoustical Society of America, 53, 1270-1287.

BeRLINER, J. E., DURLACH, N. I., \& BRAIDA, L. D. (1977). Intensity perception: VII. Further data on roving-level discrimination and the resolution and bias edge effects. Journal of the Acoustical Society of America, 61, 1577-1585.

BRAIDA, L. D., \& DURLACH, N. I. (1972). Intensity perception: II. Resolution in one-interval paradigms. Journal of the Acoustical Society of America, 51, 483-502.

Braida, L. D., Lim, J. S., Berliner, J. E., Durlach, N. I., RabiNOWITZ, W. M., \& PURKs, S. R. (1984). Intensity perception: XIII. Perceptual anchor model of context-coding. Journal of the Acoustical Society of America, 76, 722-731.

CoHEN, A. L., \& Nosofsky, R. M. (2003). An extension of the exemplarbased random-walk model to separable-dimension stimuli. Journal of Mathematical Psychology, 47, 150-165.

Durlach, N. I., \& Braida, L. D. (1969). Intensity perception: I. Preliminary theory of intensity resolution. Journal of the Acoustical Society of America, 46, 372-383.

HeAthCote, A., PoPIEL, A. J., \& Mewhort, D. J. K. (1991). Analysis of response time distributions: An example using the Stroop Task. Psychological Bulletin, 109, 340-347.

HoCKLEY, W. E. (1984). Analysis of response time distributions in the study of cognitive processes. Journal of Experimental Psychology: Learning, Memory, \& Cognition, 6, 598-615.

Hockley, W. E., \& Corballis, M. C. (1982). Test of serial scanning in item recognition. Canadian Journal of Psychology, 36, 189-212.

Holland, M. K., \& Lockhead, G. R. (1968). Sequential effects in absolute judgments of loudness. Perception \& Psychophysics, 3, 409414.

LACOUTURE, Y. (1995). Expanding MEL response box to accommodate up to 16 external buttons. Behavior Research Methods, Instruments, \& Computers, 27, 506-511.

LACOUTURE, Y. (1997). Bow, range, and sequential effects in absolute identification: A response-time analysis. Psychological Research, 60, 121-123.

Lacouture, Y., \& Lacerte, D. (1997). Stimulus modality and stimulus-response compatibility in absolute identification. Canadian Journal of Experimental Psychology, 51, 165-170.

LaCouture, Y., LI, S.-C., \& MARLEY, A. A. J. (1998). The roles of stimulus and response set size in the identification and categorization of unidimensional stimuli. Australian Journal of Psychology, 50, $165-$ 174.

Lacouture, Y., \& Marley, A. A. J. (1991). A connectionist model of choice and reaction time in absolute identification. Connection Science, 3, 401-433.

Lacouture, Y., \& Marley, A. A. J. (1995). A mapping model of the bow effect in absolute identification. Journal of Mathematical Psychology, 39, 383-395.

LaCOUTURe, Y., \& MARLey, A. A. J. (2001). Full response time distributions in absolute identification modeled via leaky competing decision processes. In E. Sommerfeld, R. Kompass, \& T. Lachmann (Eds.), Proceedings of the Seventeenth Annual Meeting of the International Society for Psychophysics (pp. 136-141). Leipzig: International Society for Psychophysics.

LAMBERTS, K. (2000). Information-accumulation theory of speeded categorization. Psychological Review, 107, 227-260.

LAMING, D. R. J. (1984). The relativity of "absolute" judgment. British Journal of Mathematical \& Statistical Psychology, 37, 152-183. 
LAming, D. R. J. (1997). The measurement of sensation. Oxford: Oxford University Press.

LockHEAd, G. R. (1984). Sequential predictors of choice in psychophysical tasks. In S. Kornblum \& J. Requin (Eds.), Preparatory states and processes (pp. 27-47). Hillsdale, NJ: Erlbaum.

LucE, R. D. (1986). Response times. New York: Oxford University Press.

Luce, R. D., Green, D. M., \& Weber, D. L. (1976). Attention bands in absolute identification. Perception \& Psychophysics, 20, 49-54.

Luce, R. D., Nosofsky, R. M., Green, D. M., \& Smith, A. F. (1982). The bow and sequential effects in absolute identification. Perception \& Psychophysics, 32, 397-408.

MCCLELland, J. L. (1979). On the time relations of mental processes: An examination of systems of processes in cascade. Psychological Review, 86, 287-330.

McGILL, W. J. (1963). Stochastic latency mechanisms. In R. D. Luce, R. R. Bush, \& E. Galanter (Eds.), Handbook of mathematical psychology (Vol. 1, pp. 309-360). New York: Wiley.

MiLleR, G. A. (1956). The magical number seven, plus or minus two: Some limits on our capacity for information processing. Psychological Review, 63, 81-97.

MORI, S. (1989). A limited-capacity response process in absolute identification. Perception \& Psychophysics, 46, 167-173.

Nosofsky, R. M. (1997). An exemplar-based random-walk model of speeded categorization and absolute judgment. In A. A. J. Marley (Ed.), Choice, decision, and measurement (pp. 347-365). Hillsdale, NJ: Erlbaum.

Nosofsky, R. M., \& Palmeri, T. J. (1997). Comparing exemplarretrieval and decision-bound models of speeded perceptual classification. Perception \& Psychophysics, 59, 1027-1048.

Parker, S., Murphy, D. R., \& SChNeIDER, B. A. (2002). Top-down gain control in the auditory system: Evidence from identification and discrimination experiments. Perception \& Psychophysics, 64, 598615.

Ratcliff, R., \& Murdock, B. B. (1976). Retrieval processes in recognition memory. Psychological Review, 83, 190-214.

Rouder, J. N., Morey, R. D., Cowan, N., \& Pfaltz, M. (2004). Learning in a unidimensional absolute identification task. Psychonomic Bulletin \& Review, 11, 938-944.

SCHNEIDER, W. (1988). Micro Experimental Laboratory: An integrated system for IBM PC compatibles. Behavior Research Methods, Instruments, \& Computers, 20, 206-217.

ShIFfrin, R. M., \& Nosofsky, R. M. (1994). Seven plus or minus two: A commentary on capacity limitations. Psychological Review, 101, 357-361.

TeGHTSOONIan, R. (1971). On the exponents in Stevens' law and the constant in Ekman's law. Psychological Review, 86, 3-27.

Treisman, M., \& Williams, T. C. (1984). A theory of criterion setting with an application to sequential dependencies. Psychological Review, 91, 68-111.

Usher, M., \& McClelland, J. L. (2001). The time course of perceptual choice: The leaky, competing accumulator model. Psychological Review, 108, 550-592.

VAN ZANDT, T. (2000). How to fit a response time distribution. Psychonomic Bulletin \& Review, 7, 424-465.

WARD, L. M. (1979). Stimulus information and sequential dependencies in magnitude estimation and cross-modality matching. Journal of Experimental Psychology: Human Perception \& Performance, $\mathbf{5}_{2}$ 444-459.

WARD, L. M., \& LocKHEAD, G. R. (1970). Sequential effects and memory in category judgments. Journal of Experimental Psychology, 84, 27-34.

WARD, L. M., \& LocKHEAD, G. R. (1971). Response system processes in absolute judgment. Perception \& Psychophysics, 9, 73-78.

Weber, D. L., Green, D. M., \& Luce, R. D. (1977). Effects of practice and distribution of auditory signals on absolute identification. Perception \& Psychophysics, 22, 223-231.

Yuille, A. L., \& GeIGER, D. (1995). Winner-take-all mechanisms. In M. A. Arbib (Ed.), The handbook of brain theory and neural networks (pp. 1056-1060). Cambridge, MA: MIT Press, Bradford Books.

\section{NOTES}

1. The required "precise value" is determined by the spacing between adjacent stimuli on each dimension. Different representations of the information (e.g., exemplar vs. general recognition theory [GRT] modelssee the Summary and Future Directions section) may assume different ways of combining the information on the two dimensions.

2. All the theory and data in this article concern the unidimensional case, so that qualifier is dropped in the remainder of the article.

3. The stimuli in our experiments are, to within the accuracy of the screen resolution, equally spaced logarithmically. When Weber's law holds, which is the case with line length in the range we study, stimuli spaced logarithmically are likely to be equally discriminable and, therefore, equally spaced along the decision axis in an identification task.

4. There is a conceptual distinction between the assumption that the accumulator contents are reset to zero at the beginning of each trial and the fact (or assumption) that the effect of a stimulus starts at zero at the beginning of a trial. See the Summary and Future Directions section for further discussion.

5. Although $\Delta$ can be absorbed into the original Equation A3 by adjustment of the various parameters, we choose to keep $\Delta$ separate for explicit control of the simulated time steps.

6. For this reason, in the remainder of the article, we let mean $R T$ stand for "mean response time for correct responses." Later, we briefly summarize and analyze the error data. 


\section{APPENDIX}

The Leaky Competing Accumulator Decision Process

There are $N$ decision units (accumulators). Using Usher and McClelland's (2001) notation, for a given input $i, i=1, \ldots, N$, let $x_{i}, i=1, \ldots, N$, be the activation signal received by accumulator $i$, and let $f_{i}$ be the "firing" rate of accumulator $i$ such that $f_{i}=x_{i}$ for $x_{i}>0$ and $f_{i}=0$ for $x_{i}<0$. We assume that the first accumulator to reach a stable state (see below) triggers the specific overt response associated with it and that the RT is the time taken for the relevant (first) process to reach a stable state. For each accumulator, the change in activation $d x_{i}$ is given by

$$
d x_{i}=\left[I_{i}-\lambda x_{i}\right] \frac{d t}{\tau}+\xi_{i} \sqrt{\frac{d t}{\tau}}
$$

where $(d t) / \tau$ is a time scale, $\xi_{i}$ is a Gaussian noise term with zero mean and nonzero variance, $I_{i}$ is the input to accumulator $i$, and $\lambda$ is a decay rate. Input $I_{i}$ can be decomposed into three components: an external source, which in our case is the output from the mapping process; a recurrent connection from the unit to itself that provides a self-amplification signal; and an inhibitory signal coming from the other accumulators. ${ }^{\mathrm{A} 1}$ Equation $\mathrm{A} 1$ is then expanded into the form

$$
d x_{i}=\left[\rho_{i}-\lambda x_{i}+\alpha f_{i}-\beta \sum_{i^{\prime} \neq i} f_{i^{\prime}}\right] \frac{d t}{\tau}+\xi_{i} \sqrt{\frac{d t}{\tau}},
$$

where the change in activation for accumulator $i$ depends on the external input with value $\rho_{I}$; the leakage with parameter $\lambda$; the self-amplification with parameter $\alpha$; and the inhibitory signal from the other accumulators with parameter $\beta$. For $x_{i}>0$, the equation is linear and we can replace the value $(\alpha-\lambda)$ with a net leakage parameter $l$. For $l>0$, activation decays, and for $l<0$ activation self-amplifies. For reasons discussed in Usher and McClelland, we can replace the firing rate $f_{i}$ in Equation A2 by activation levels $x_{i}$, and truncate $x_{i}$ at zero. We then have

$$
\begin{aligned}
& d x_{i}=\left[\rho_{i}-l x_{i}-\beta \sum_{i^{\prime} \neq i} x_{i^{\prime}}\right] \frac{d t}{\tau}+\xi_{i} \sqrt{\frac{d t}{\tau}}, \\
& \text { with } \quad x_{i} \rightarrow \max \left(x_{i}, 0\right) .
\end{aligned}
$$

Note the nonlinearity introduced in the second line, where the value of $x_{i}$ is set to zero if $x_{i} \leq 0$. Equation A3 gives a close approximation to Equation A2 with parameter values in the ranges we use; also see Appendix A of Usher and McClelland (2001) for mathematical arguments on this point. Usher and McClelland applied this leaky competing accumulator process to two-alternative tasks; we apply it to $\mathrm{N}$-alternative absolute identification and binary categorization.

\section{NOTE}

A1. In Usher and McClelland (2001), the input to accumulator $i$ corresponds to the value of stimulus $i$. In our application, the input to accumulator $j, j=1, \ldots, N$, for a given stimulus $x_{i}, i=1, \ldots, N$, is the output activation $O(j \mid i)$ of our mapping model.

(Manuscript received December 19, 2002; revision accepted for publication March 29, 2004.) 